\title{
Stochastic Topology Design Optimization for Continuous Elastic Materials.
}

\author{
Miguel Carrasco ${ }^{\mathrm{a}}$, Benjamin Ivorra ${ }^{\mathrm{b}, *}$, Angel Manuel Ramos ${ }^{\mathrm{b}}$ \\ ${ }^{a}$ Facultad de Ingeniería y Ciencias Aplicadas, Universidad de los Andes, Mons. Álvaro \\ del Portillo 12.455, Las Condes, Santiago, Chile \\ ${ }^{b}$ Departamento de Matemática Aplicada 83 Instituto de Matemática Interdisciplinar, \\ Universidad Complutense de Madrid, Plaza de Ciencias, 3, 28040-Madrid, Spain
}

\begin{abstract}
In this paper, we develop a stochastic model for topology optimization. We find robust structures that minimize the compliance for a given main load having a stochastic behavior. We propose a model that takes into account the expected value of the compliance and its variance. We show that, similarly to the case of truss structures, these values can be computed with an equivalent deterministic approach and the stochastic model can be transformed into a nonlinear programming problem, reducing the complexity of this kind of problems. First, we obtain an explicit expression (at the continuous level) of the expected compliance and its variance, then we consider a numerical discretization (by using a finite element method) of this expression and finally we use an optimization algorithm. This approach allows to solve design problems which include point, surface or volume loads with dependent or independent perturbations. We check the capacity of our formulation to generate structures that are robust to main loads and their perturbations by considering several $2 \mathrm{D}$ and $3 \mathrm{D}$ numerical examples. To this end, we analyze the behavior of our model by studying the impact on the optimized solutions of the expected-compliance and variance weight coefficients, the laws used to describe the random loads, the variance of the perturbations and the dependence/independence of the perturbations. Then, the results are compared with similar ones found in the literature for a different modeling
\end{abstract}

\footnotetext{
*Corresponding author

Email addresses: micarrasco@uandes.cl (Miguel Carrasco), ivorra@mat.ucm.es (Benjamin Ivorra ), angel@mat.ucm.es (Angel Manuel Ramos)
} 
approach.

Keywords: Topology optimization; Structural optimization; Stochastic programming; Finite element method.

2000 MSC: 35Q74; 74B05; 74P05; 74S60.

\section{Introduction}

Let us consider an open set $\Omega \subset \mathbb{R}^{d}$, where $d$ is 2 for planar structures or 3 for three-dimensional bodies. The set $\Omega$ represents a body that we assume is made of an isotropic, homogeneous and linear elastic material. The boundary of $\Omega$ is denoted here by $\partial \Omega=\Gamma_{u} \cup \Gamma_{g}$ with $\Gamma_{u} \cap \Gamma_{g}=\emptyset$. In this setting, $\Gamma_{u}$ corresponds to the part of the boundary of $\Omega$ where the displacements of the body are not allowed. We assume that external forces $f$ and $g$ are applied to $\Omega$ and $\Gamma_{g}$, respectively. A graphical representation of $\Omega$ is given in Figure 1 .

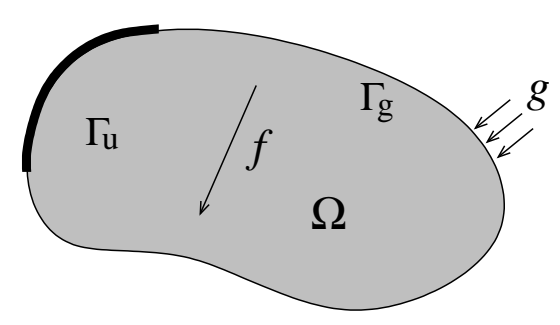

Figure 1: Representation of the elastic homogeneous body $\Omega$ and the considered external forces $f$ and $g$.

The displacements can be computed (see e.g. [1]) by solving the following system of partial differential equations:

$$
\left\{\begin{aligned}
-\operatorname{div}(K e(u)) & =f, & & \text { in } \Omega, \\
u & =0, & & \text { on } \Gamma_{u}, \\
(K e(u)) \cdot \hat{n} & =g, & & \text { on } \Gamma_{g},
\end{aligned}\right.
$$

where $u: \Omega \rightarrow \mathbb{R}^{d}$ is the vector of displacements, $e(u)=\frac{1}{2}\left(\nabla u+\nabla u^{t}\right)$ denotes the linearized strain tensor, $K$ is the fourth-order material elasticity tensor, $\operatorname{div}(\cdot)$ is the divergence of a tensor field and $\hat{n}$ is the outward unit normal vector on the boundary of the domain. We suppose that $K \in M$, which is a set of admissible stiffness tensors, related to the admissible materials we 
might use. Typically (see $[2,3]$ ), the set of admissible tensor $M$ is a subset of

$$
\hat{M}=\left\{\eta(x) K^{0} \mid \int_{\Omega} \eta(x) \mathrm{d} x \leq V_{\max } \text { and } \eta: \Omega \rightarrow\left[\eta_{\min }, \eta_{\max }\right]\right\},
$$

where $V_{\max }$ is the maximum amount of material that is allocated; $\eta(x)$ is the density of material at point $x \in \Omega ; \eta_{\min }>0, \eta_{\max }$ the maximum density we may use; and $K^{0}$ the fourth-order tensor of a linear elastic isotropic reference material. Additionally, other constraints should be considered in order to obtain physically realizable structures. For example, we would like to avoid structures with intermediate density zones (i.e., $\eta(x) \in] \eta_{\min }, \eta_{\max }[$ ), and microstructures with periodic variation density. For practical reasons, these constraints are not detailed in the continuous definition of $M$ but they will be taken into account in its numerical implementation (see Section 3).

In the following, we assume that $f \in L^{2}(\Omega)^{d}$ and $g \in L^{2}\left(\Gamma_{g}\right)^{d}$ although less regular external forces can be also considered (see Remark 1 below)

Let us define $H=\left\{u \in\left[H^{1}(\Omega)\right]^{d}|u|_{\Gamma_{u}}=0\right\}$, where the space $H^{1}(\Omega)$ is the well-known Sobolev space of functions that are in $L^{2}(\Omega)$ with the first derivatives (in the sense of distributions) in $L^{2}(\Omega)$. For a given material and its corresponding stiffness tensor $K \in M$, following $[2,4]$ we define the bilinear functional $A_{K}: H \times H \rightarrow \mathbb{R}$ by

$$
A_{K}(u, v):=\int_{\Omega} e(u): K e(v) \mathrm{d} x,
$$

where $e(u): K e(v)$ denotes the tensor product given by

$$
e(u): K e(v):=\sum_{i, j, k, l=1}^{d} K_{i j k l} e_{i j}(u) e_{k l}(v) .
$$

We recall that a weak solution of system (1) is a vector $u \in H$ satisfying

$$
A_{K}(u, v)=\int_{\Omega} f \cdot v \mathrm{~d} x+\int_{\Gamma_{g}} g \cdot v \mathrm{~d} x \quad \forall v \in H .
$$

We note that, under suitable conditions on the data (according to Korn's inequality and the Lax-Milgram Lemma [5, 6]), Problem (1) has a unique weak solution (see [7] for more details).

Remark 1. In this work it is possible to include functions less regular than $f \in L^{2}(\Omega)$ or $g \in L^{2}\left(\Gamma_{g}\right)$ with a suitable alternative weak formulation instead 
of (4). An example of force $f \notin L^{2}(\Omega)$ that is typically considered is a point wise force $f=\bar{f} \delta(x-a)$, where $\bar{f} \in \mathbb{R}^{d}$ and $a \in \bar{\Omega}$. In this case, $\int_{\Omega} f \cdot v \mathrm{~d} x=\bar{f} v(a)$. Nevertheless, when computing an approximated solution by using the Finite Element Method (see Section 3) $f$ and $g$ are usually approximated by functions in $L^{2}(\Omega)^{d}$ and $L^{2}\left(\Gamma_{g}\right)^{d}$, respectively, and (4) can be used again, becoming a linear system in finite dimension.

For the sake of simplicity and without loss of generality, in the following, we only develop the case when $g=0$. To recover the general case, the reader should replace $\int_{\Omega} f \cdot v \mathrm{~d} x$ by $\int_{\Omega} f \cdot v \mathrm{~d} x+\int_{\Gamma_{g}} g \cdot v \mathrm{~d} x$, keeping in mind that the integral notation should be changed by suitable duality products for cases with less regular forces.

We note that, due to the symmetry of operator $A_{K}(\cdot, \cdot)$, equation (4) (with $g=0$ ) above can be seen as the optimality condition of the following problem

$$
\frac{1}{2} \int_{\Omega} f \cdot u \mathrm{~d} x=\max _{v \in H}\left\{\int_{\Omega} f \cdot v \mathrm{~d} x-\frac{1}{2} A_{K}(v, v)\right\} .
$$

The optimal value of the problem above is called compliance (see e.g. [2]) and can be seen as a measure of the global stiffness of a given material. Under this setting the well known minimum compliance topology design problem corresponds to (see $[3,2])$

$$
\begin{aligned}
& \min _{K \in M}\left\{\frac{1}{2} \int_{\Omega} f \cdot u \mathrm{~d} x, \text { where } u \in H\right. \text { satisfies: } \\
& \left.\qquad A_{K}(u, v)=\int_{\Omega} f \cdot v \mathrm{~d} x \forall v \in H\right\} .
\end{aligned}
$$

Our main purpose is to find an optimal material in the set $M$ (minimum compliance) when the external load force has a stochastic behavior. In an analogous way to previous stochastic results for truss optimization (see $[8,9,10]$ and the next section), we assume that the external load force $f$ is perturbed by a $L^{2}(\Omega)^{d}$-valued random variable $\xi: \mathcal{B} \rightarrow L^{2}(\Omega)^{d}$, where $(\mathcal{B}, \mathcal{F}, \mathbb{P})$ is a probability space ${ }^{1}$, with expected value

$$
\mathbb{E}(\xi)=\int_{\mathcal{B}} \xi \mathrm{d} \mathbb{P}=\int_{\mathcal{B}} \xi(\omega) \mathbb{P}(\mathrm{d} \omega)=0 \in L^{2}(\Omega)^{d} .
$$

\footnotetext{
${ }^{1}$ With this notation $\mathcal{B}$ is the sample space, $\mathcal{F}$ denotes the $\sigma$-field and $\mathbb{P}$ its probability measure.
} 
Remark 2. Similarly, when $g \neq 0$, the surface load is perturbed by a $L^{2}\left(\Gamma_{g}\right)^{d}$ - valued random variable $\xi: \mathcal{B} \rightarrow L^{2}\left(\Gamma_{g}\right)^{d}$ with $\mathbb{E}(\xi)=0$.

For a given material $K \in M$ and a random perturbation $\xi$ of the external load, we have the corresponding compliance

$$
\begin{array}{r}
\Psi(\xi, K)=\int_{\Omega}(f+\xi) \cdot u \mathrm{~d} x, \text { where } u \in H \text { satisfies: } \\
A_{K}(u, v)=\int_{\Omega}(f+\xi) \cdot v \mathrm{~d} x \quad \text { for all } v \text { in } H .
\end{array}
$$

Following $[8,2,4]$, we state the stochastic topology design problem as

$$
\min _{K \in M} \mathbb{E}[\Psi(\xi, K)]
$$

where the expected value of the random variable $\Psi(\xi, K)$ is given by

$$
\mathbb{E}(\Psi(\xi, K))=\int_{\mathcal{B}} \Psi(\xi(\omega), K) \mathbb{P}(\mathrm{d} \omega) .
$$

We note that, when the support of the probability distribution is the singleton $\{0\}$, i.e. $\mathcal{B}=\{\xi=0\}$ and $\mathbb{P}\{\xi=0\}=1$, Problem (8) corresponds to the minimum compliance design model (6). Also when the probability distribution has finite support we obtain the well known multiload model (see $[2,3])$. We recall that the multiload model was proposed in order to take into account several loading scenarios or loads that are applied in different instant of time. This model corresponds to the minimization of a weighted average of the compliance. More precisely, if we consider the functions $f_{i}: \Omega \rightarrow \mathbb{R}^{d}$, for $i=1, \ldots, k$, and the functional $A_{K}(\cdot, \cdot)$ defined in eq. (4), the multiload model is given by

$$
\begin{array}{r}
\min _{K \in M}\left\{\sum_{i} w_{i} \int_{\Omega} f_{i} \cdot u_{i} \mathrm{~d} x, \quad \text { where } u_{i} \in H\right. \text { satisfies: } \\
\left.A_{K}\left(u_{i}, v\right)=\int_{\Omega} f_{i} \cdot v \mathrm{~d} x, \text { for all } v \text { in } H\right\} .
\end{array}
$$

Taking the discrete probability law given by $\mathcal{B}=\left\{\xi=f_{1}, \ldots, \xi=f_{k}\right\}$ and $f=0$, defining the weights as $w_{i}:=\mathbb{P}\left\{\xi=f_{i}\right\}$ (the probability that discrete variable $\xi$ take the value $f_{i}$ ), we get that the previous problem is a particular case of (8), and therefore, the proposed model extends classical formulations. 
In [11] formulation (8) was studied for some choices of the random variable $\xi$ and good results were obtained. From a theoretical point of view, it is showed that the expected value can be computed with an equivalent deterministic approach which reduces drastically the computational cost of computing a solution of the stochastic Problem (8) (see Section 2 for a summary of these results). Numerical experiments have been carried out for some particular benchmarks using the expected compliance formulation (8). Recently, a new approach based on numerical and Monte Carlo methods has been proposed by Zhao and Wang (see [12]) in order to obtain robust structures under load uncertainty. In other direction, Allaire (see [13]) proposes a deterministic method for optimizing a structure with respect to its worst possible behavior. Similarly to the case of trusses (see [10]) this approach provides solutions which are robust under load perturbations, but for some particular scenarios it may happen that the value of the compliance is too large and this model is no suitable. In order to avoid this situation we propose the following alternative.

$$
\min _{K \in M}\left\{\frac{\alpha}{\mathbb{E}_{0}} \mathbb{E}[\Psi(\xi, K)]+\frac{\beta}{\operatorname{Var}_{0}} \operatorname{Var}[\Psi(\xi, K)]\right\},
$$

where Var is the variance of the random variable $\Psi(\xi, K) ; \alpha, \beta \in[0,1]$ are weight coefficients; and $\mathbb{E}_{0}, \operatorname{Var}_{0} \in \mathbb{R}$ denote reference expected compliance and variance values used to normalize $\mathbb{E}[\Psi(\xi, K)]$ and $\operatorname{Var}[\Psi(\xi, K)]$, respectively. Problems similar to (10) have been studied in [14, 15, 16, 17, 18, 19] applying stochasticity at the nodes of a finite element discretization of the considered infinite dimensional optimization problem. We show here that more general problems can be tackled when working directly with the continuous weak form of (1), in a way similar to [4] and [20], but without using Monte-Carlo methods or other approximation techniques of the random loads.

We point out that in this work we first obtain an explicit expression of $\mathbb{E}[\Psi(\xi, K)]$ and $\operatorname{Var}[\Psi(\xi, K)]$ and then discretize it. This allows us to consider design problems including general point, surface or volume loads with dependent or independent perturbations. In other works (see, for instance $[14,15,16,17,18])$ they first discretize $\Psi(\xi, K)$ and then compute the expected and variance values of the discretized problem, being therefore limited by the chosen discretization.

In Section 2 we show the general mathematical framework for solving Problem (10). More precisely, we give explicit expressions to the expected 
value and the variance of the functional $\Psi$ mentioned above, and show that the stochastic model proposed here can be transformed into a equivalent nonlinear programming problem. In Section 3, we detail the numerical methods used to solve this nonlinear programming problem. Finally, in Section 4, we illustrate the interest of formulation (10) by considering several $2 \mathrm{D}$ and $3 \mathrm{D}$ benchmark problems. Therefore, after describing the considered numerical implementation, we introduce four set of benchmark problems to analyze the impact (for instance, variations on geometric or compliance thresholds) on the solutions of (i) the coefficients $(\alpha ; \beta)$, (ii) the law used to describe the random loads perturbations, (iii) the loads perturbation variance and (iv) the dependence or independence of the loads perturbations. Indeed, those four model characteristics were observed in previous literature $[2,8,10,15,16]$, considering a different modeling approach, to have an important influence on the characteristics of the solution.

\section{Variance-expected compliance approach for topology optimiza- tion}

\subsection{Variance-expected approach}

In this section, we study the stochastic topology design problem (10) proposed in Section (1). We show that this problem can be transformed into a multiload like problem in which the loading scenarios are related to the variance of random loads applied to $\Omega$.

Firstly, we consider the stochastic topology optimization problem (8). In the following, we will consider the set $\left\{P_{i}\right\}_{i=1}^{\infty} \subset L^{2}(\Omega)^{d}$, corresponding to directions of perturbation of the main force $f \in L^{2}(\Omega)$. As said in Remark 1 , spaces less regular that $L^{2}(\Omega)^{d}$ can be also considered.

Theorem 1 below gives an explicit expression of Problem (8) which can be directly evaluated. Therefore, a Monte-Carlo algorithm (see [21]), which is usually numerically expensive, is not necessary to approximate the value of $\mathbb{E}[\Psi(\xi, K)]$.

In the following we define the inverse functional $G:\left[L^{2}(\Omega)\right]^{d} \rightarrow H$ by $G(f)=u$, where $u$ is the (unique) weak solution of (1) or, equivalently, (4) or (5). Then, for a given random perturbation $\xi \in L^{2}(\Omega)^{d}$ and a tensor $K \in M$ we have that

$$
\Psi(\xi, K)=\int_{\Omega}(f+\xi) \cdot G(f+\xi) \mathrm{d} x .
$$


Theorem 1. With the notation introduced above let us consider $\xi: \mathcal{B} \rightarrow$ $L^{2}(\Omega)^{d}$ be a random load, which in terms of the directions $\left\{P_{i}\right\}_{i=1}^{\infty}$ is written as $\xi(\omega)=\sum_{i=1}^{\infty} \varepsilon_{i}(\omega) P_{i}$, where $\left\{\varepsilon_{i}\right\}_{i=1}^{\infty}$ are random variables such that $\mathbb{E}\left(\varepsilon_{i}\right)=$ 0 , and $\operatorname{Var}\left(\varepsilon_{i}\right)=\sigma_{i}^{2}$. Then

$$
\begin{aligned}
& \mathbb{E}(\Psi(\xi, K))=\int_{\Omega} f \cdot u \mathrm{~d} x+\sum_{i, j=1}^{+\infty} \mathbb{E}\left(\varepsilon_{i} \varepsilon_{j}\right) \int_{\Omega} P_{i} \cdot U_{j} \mathrm{~d} x \\
& \text { where } u \in H \text { such that } A_{K}(u, v)=\int_{\Omega} f \cdot v \mathrm{~d} x, \forall v \in H \\
& U_{i} \in H \text { such that } A_{K}\left(U_{i}, v\right)=\int_{\Omega} P_{i} \cdot v \mathrm{~d} x, \forall v \in H, \forall i \in \mathbb{N} .
\end{aligned}
$$

Proof: By the linearity of the integral and the operator $G(\cdot)$, we obtain

$$
\begin{aligned}
\Psi(\xi, K)=\int_{\Omega} f \cdot G(f) \mathrm{d} x & +\sum_{i=1}^{+\infty} \varepsilon_{i} \int_{\Omega} f \cdot G\left(P_{i}\right) \mathrm{d} x \\
& +\sum_{i=1}^{+\infty} \varepsilon_{i} \int_{\Omega} P_{i} \cdot G(f) \mathrm{d} x+\sum_{i, j=1}^{+\infty} \varepsilon_{i} \varepsilon_{j} \int_{\Omega} P_{i} \cdot G\left(P_{j}\right) \mathrm{d} x
\end{aligned}
$$

The conclusion follows by taking expected value to the expression above and using that $\mathbb{E}\left(\varepsilon_{i}\right)=0$.

Remark 3. We note that the expression (11) can be written equivalently as

$$
\mathbb{E}(\Psi(\xi, K))=\int_{\Omega} f \cdot u \mathrm{~d} x+\sum_{i \neq j}^{+\infty} \mathbb{E}\left(\varepsilon_{i} \varepsilon_{j}\right) \int_{\Omega} P_{i} \cdot U_{j} \mathrm{~d} x+\sum_{i=1}^{+\infty} \sigma_{i}^{2} \int_{\Omega} P_{i} \cdot U_{i} \mathrm{~d} x
$$

and the second term of the right hand side of (15) disappears when the random variables $\varepsilon_{i}$ are independent (since $\mathbb{E}\left(\varepsilon_{i} \varepsilon_{j}\right)=0$ if $i \neq j$ ).

Remark 4. It can be proved that $L^{2}(\Omega)^{d}$ (also $L^{2}\left(\Gamma_{g}\right)^{d}$ and many other spaces suitable for cases with $f$ and $g$ less regular) is a separable Hilbert space and, therefore, there exist a Hilbert basis $\left\{P_{i}\right\}_{i=1}^{\infty} \subset L^{2}(\Omega)^{d}$. So that, for any $\xi \in L^{2}(\Omega)^{d}$ there exists $\left\{\varepsilon_{i}\right\}_{i=1}^{\infty} \subset \mathbb{R}$ such that $\xi=\sum_{i=1}^{\infty} \varepsilon_{i} P_{i}$. For 
arbitrary domains $\Omega$ finding such a base is not easy, but for simple domains $\Omega$ as intervals or rectangles, a Hilbert base for spaces as $L^{2}(\Omega)$ or $H^{1}(\Omega)$ can be easily obtained in terms, for instance, of sinusoidal functions. Results about this and some examples can be found in [22].

In order to avoid scenarios with too large values of the compliance we consider Problem (10) instead of (8). We recall that the variance of $\Psi(\xi, K)$ is computed by using the well known formula

$$
\operatorname{Var}[\Psi(\xi, K)]=\mathbb{E}\left[\Psi(\xi, K)^{2}\right]-\mathbb{E}[\Psi(\xi, K)]^{2} .
$$

We can give an explicit formula of (16) for some examples of perturbation function, as the next theorem shows.

Theorem 2. Let us consider $\xi=\sum_{i=1}^{+\infty} \varepsilon_{i} P_{i} \in\left[L^{2}(\Omega)\right]^{d}$, where $P_{i} \in\left[L^{2}(\Omega)\right]^{d}$ for $i \in \mathbb{N}$ and $\varepsilon_{i}$ are real random variables that satisfy $\mathbb{E}\left(\varepsilon_{i}\right)=0$ for $i \in$ $\mathbb{N}$. The value $\operatorname{Var}[\Psi(\xi, K)]$ can be computed explicitly by using the following identities:

$$
\begin{aligned}
& \mathbb{E}\left[\left(\int_{\Omega} f \cdot G(\xi) \mathrm{d} x\right)^{2}\right]=\sum_{i, j=1}^{+\infty} \mathbb{E}\left(\varepsilon_{i} \varepsilon_{j}\right) \int_{\Omega} f \cdot G\left(P_{i}\right) \mathrm{d} x \int_{\Omega} f \cdot G\left(P_{j}\right) \mathrm{d} x, \\
& \mathbb{E}\left[\left(\int_{\Omega} \xi \cdot G(f) \mathrm{d} x\right)^{2}\right]=\sum_{i, j=1}^{+\infty} \mathbb{E}\left(\varepsilon_{i} \varepsilon_{j}\right) \int_{\Omega} P_{i} \cdot G(f) \mathrm{d} x \int_{\Omega} P_{j} \cdot G(f) \mathrm{d} x, \\
& \mathbb{E}\left[\int_{\Omega} f \cdot G(\xi) \mathrm{d} x \int_{\Omega} \xi \cdot G(f) \mathrm{d} x\right]= \\
& \qquad \sum_{i, j=1}^{+\infty} \mathbb{E}\left(\varepsilon_{i} \varepsilon_{j}\right) \int_{\Omega} f \cdot G\left(P_{i}\right) \mathrm{d} x \int_{\Omega} P_{j} \cdot G(f) \mathrm{d} x \\
& \mathbb{E}\left[\int_{\Omega} f \cdot G(\xi) \mathrm{d} x \int_{\Omega} \xi \cdot G(\xi) \mathrm{d} x\right]= \\
& \sum_{i, j, k=1}^{+\infty} \mathbb{E}\left(\varepsilon_{i} \varepsilon_{j} \varepsilon_{k}\right) \int_{\Omega} f \cdot G\left(P_{i}\right) \mathrm{d} x \int_{\Omega} P_{j} \cdot G\left(P_{k}\right) \mathrm{d} x
\end{aligned}
$$




$$
\begin{gathered}
\mathbb{E}\left[\int_{\Omega} \xi \cdot G(f) \mathrm{d} x \int_{\Omega} \xi \cdot G(\xi) \mathrm{d} x\right]= \\
\sum_{i, j, k=1}^{+\infty} \mathbb{E}\left(\varepsilon_{i} \varepsilon_{j} \varepsilon_{k}\right) \int_{\Omega} P_{i} \cdot G(f) \mathrm{d} x \int_{\Omega} P_{j} \cdot G\left(P_{k}\right) \mathrm{d} x, \\
\mathbb{E}\left[\left(\int_{\Omega} \xi \cdot G(\xi) \mathrm{d} x\right)^{2}\right]= \\
\sum_{i, j, k, l=1}^{+\infty} \mathbb{E}\left(\varepsilon_{i} \varepsilon_{j} \varepsilon_{k} \varepsilon_{l}\right) \int_{\Omega} P_{i} \cdot G\left(P_{j}\right) \mathrm{d} x \int_{\Omega} P_{k} \cdot G\left(P_{l}\right) \mathrm{d} x .
\end{gathered}
$$

Proof: Equations (17)-(22) can be computed directly by using the linearity of operator $G(\cdot)$ and the properties of the expected value. Additionally, by the linearity of operator $G(\cdot)$, we obtain

$$
\Psi(\xi, K)=\int_{\Omega} f \cdot G(f) \mathrm{d} x+\int_{\Omega} f \cdot G(\xi) \mathrm{d} x+\int_{\Omega} \xi \cdot G(f) \mathrm{d} x+\int_{\Omega} \xi \cdot G(\xi) \mathrm{d} x
$$

Therefore, from (16), we obtain an expression for the variance of the compliance by using identities (17)-(22).

As we mentioned in the introduction this approach can be applied for functions belonging to a more general functional space that include for example, surface loads or Dirac- $\delta$ functions. This issue and the following results are relevant for the numerical experiences given in the next section.

We recall that, in the particular case in which $\varepsilon_{i}$ are independent random variables, with $\varepsilon_{i} \sim \mathcal{N}\left(0, \sigma_{i}^{2}\right)$ for $i \in \mathbb{N}$ we get

$$
\begin{aligned}
& \mathbb{E}\left(\varepsilon_{i} \varepsilon_{j}\right)=\left\{\begin{array}{ll}
\sigma_{i}^{2} & \text { if } i=j \\
0 & \text { if } i \neq j
\end{array}, \quad \mathbb{E}\left(\varepsilon_{i} \varepsilon_{j} \varepsilon_{k}\right)=0, \forall i, j, k \in \mathbb{N},\right. \\
& \mathbb{E}\left(\varepsilon_{i} \varepsilon_{j} \varepsilon_{k} \varepsilon_{l}\right)= \begin{cases}3 \sigma_{i}^{4} & \text { if } i=j=k=l, \\
\sigma_{i}^{2} \sigma_{j}^{2} & \text { if } i=k, j=l \text { or } i=l, j=k \text { and } i \neq j, \\
\sigma_{i}^{2} \sigma_{k}^{2} & \text { if } i=j, k=l \text { and } i \neq k, \\
0 & \text { either case. }\end{cases}
\end{aligned}
$$

The following Corollaries shows the particular case of two perturbation functions. 
Corollary 1. Using the notation of the previous theorem, let us consider a random perturbation of $f$ given by $\xi=\varepsilon_{1} P_{1}+\varepsilon_{2} P_{2}$, where the perturbation functions $P_{1}, P_{2} \in L^{2}(\Omega)^{d}$ and $\varepsilon_{1}, \varepsilon_{2}$ are random variables, with $\varepsilon_{i} \sim$ $\mathcal{N}\left(0, \sigma_{i}^{2}\right)$. Then

$$
\begin{array}{r}
\operatorname{Var}[\Psi(\xi, K)]=\sigma_{1}^{2}\left(\int_{\Omega} f \cdot U_{1}+P_{1} \cdot u \mathrm{~d} x\right)^{2}+\sigma_{2}^{2}\left(\int_{\Omega} f \cdot U_{2}+P_{2} \cdot u \mathrm{~d} x\right)^{2} \\
+2 \cdot \sigma_{1}^{4}\left(\int_{\Omega} P_{1} \cdot U_{1} \mathrm{~d} x\right)^{2}+2 \cdot \sigma_{2}^{4}\left(\int_{\Omega} P_{2} \cdot U_{2} \mathrm{~d} x\right)^{2} \\
+\left(\mathbb{E}\left(\epsilon_{1}^{2} \epsilon_{2}^{2}\right)-\mathbb{E}^{2}\left(\epsilon_{1} \epsilon_{2}\right)\right)\left(\int_{\Omega} P_{1} \cdot U_{2}+P_{2} \cdot U_{1} \mathrm{~d} x\right)^{2} \\
+2 \cdot \mathbb{E}\left(\epsilon_{1} \epsilon_{2}\right)\left(\int_{\Omega} f \cdot U_{1}+P_{1} \cdot u \mathrm{~d} x\right)\left(\int_{\Omega} f \cdot U_{2}+P_{2} \cdot u \mathrm{~d} x\right) \\
+2 \cdot \mathbb{E}\left(\epsilon_{1} \epsilon_{2}^{2}\right)\left(\int_{\Omega} f \cdot U_{1}+P_{1} \cdot u \mathrm{~d} x\right)\left(\int_{\Omega} P_{1} \cdot U_{2}+P_{2} \cdot U_{1} \mathrm{~d} x\right) \\
+2 \cdot \mathbb{E}\left(\epsilon_{1}^{2} \epsilon_{2}\right)\left(\int_{\Omega} f \cdot U_{2}+P_{2} \cdot u \mathrm{~d} x\right)\left(\int_{\Omega} P_{1} \cdot U_{2}+P_{2} \cdot U_{1} \mathrm{~d} x\right) \\
+2 \cdot\left(\mathbb{E}\left(\epsilon_{1}^{2} \epsilon_{2}^{2}\right)-\sigma_{1}^{2} \sigma_{2}^{2}\right)\left(\int_{\Omega} P_{1} \cdot U_{1} \mathrm{~d} x\right)\left(\int_{\Omega} P_{2} \cdot U_{2} \mathrm{~d} x\right) \\
+2 \cdot\left(\mathbb{E}\left(\epsilon_{1}^{3} \epsilon_{2}\right)-\sigma_{1}^{2} \mathbb{E}\left(\epsilon_{1} \epsilon_{2}\right)\right)\left(\int_{\Omega} P_{1} \cdot U_{2}+P_{2} \cdot U_{1} \mathrm{~d} x\right)\left(\int_{\Omega} P_{1} \cdot U_{1} \mathrm{~d} x\right) \\
+2 \cdot\left(\mathbb{E}\left(\epsilon_{1} \epsilon_{2}^{3}\right)-\sigma_{2}^{2} \mathbb{E}\left(\epsilon_{1} \epsilon_{2}\right)\right)\left(\int_{\Omega} P_{1} \cdot U_{2}+P_{2} \cdot U_{1} \mathrm{~d} x\right)\left(\int_{\Omega} P_{2} \cdot U_{2} \mathrm{~d} x\right)
\end{array}
$$

where $u=G(f), U_{i}=G\left(\sigma_{i} P_{i}\right), i=1,2$. In other words,

$$
A_{K}(u, v)=\int_{\Omega} f \cdot v \mathrm{~d} x, A_{K}\left(U_{i}, v\right)=\int_{\Omega} \sigma_{i} P_{i} \cdot v \mathrm{~d} x, \forall v \in H, i=1,2 .
$$

Corollary 2. Considering the notations introduced in Corollary 1, let us consider a random perturbation of $f$ given by $\xi=\varepsilon_{1} P_{1}+\varepsilon_{2} P_{2}$, where the perturbation functions $P_{1}, P_{2} \in L^{2}(\Omega)^{d}$ and $\varepsilon_{1}, \varepsilon_{2}$ are independent random 
variables, with $\varepsilon_{i} \sim \mathcal{N}\left(0, \sigma_{i}^{2}\right)$. Then

$$
\begin{array}{r}
\operatorname{Var}[\Psi(\xi, K)]=\sigma_{1}^{2}\left(\int_{\Omega} f \cdot U_{1}+P_{1} \cdot u \mathrm{~d} x\right)^{2}+\sigma_{2}^{2}\left(\int_{\Omega} f \cdot U_{2}+P_{2} \cdot u \mathrm{~d} x\right)^{2} \\
+2 \cdot \sigma_{1}^{4}\left(\int_{\Omega} P_{1} \cdot U_{1} \mathrm{~d} x\right)^{2}+2 \cdot \sigma_{2}^{4}\left(\int_{\Omega} P_{2} \cdot U_{2} \mathrm{~d} x\right)^{2} \\
+\sigma_{1}^{2} \sigma_{2}^{2}\left(\int_{\Omega} P_{1} \cdot U_{2}+P_{2} \cdot U_{1} \mathrm{~d} x\right)^{2} .
\end{array}
$$

Proof of Corollary 1 and 2: The variance of the functional can be computed directly using Theorem 2, (23) and the linearity of the operator $G(\cdot)$.

Remark 5. Using Equations (17)-(22), Corollary 1 can be generalized to other cases. For instance, when $m \geq 2$ or random variables $\epsilon_{i}$ do not follow a normal distribution.

Remark 6. Similar problems are studied in [14, 15, 16, 17, 18, 19] to estimate uncertainty in loads at the nodes of a numerical discretization of the problem. However, the approach proposed here deals directly with the continuous (non-discretized) weak form of (1), in a way similar to that of [4] and [20], but without using Monte-Carlo methods (or other techniques) to approximate the random loads. Indeed, Theorems 1 and 2 give explicit formulas at the continuous level for the expected value and the variance of the compliance functional (7) (i.e., without needing to use previously a numerical discretization). Obviously, when applying the results presented in this work to solve numerically a particular design problem, some approximations and simplifications on the considered problem could be needed. For instance, in the case of considering an infinite number of random loads, one should approximate the problem by considering a well chosen finite number of loads obtained, for example, by using a Karhunen-Loeve expansion of the random field (see [19, 20]).

\subsection{Considered minimization problem formulation}

As mentioned previously, we are interested in solving minimization Problem (10). With the purpose of generating physically realizable optimized structures (see Section 1), we consider the so-called SIMP model (Solid Isotropic Material with Penalization; see $[3,2])$. In this model, the set of 
admissible tensors is a subset $M$ of the set $\hat{M}$, defined by (2), where the density $\eta(x)=\rho(x)^{p}, x \in \Omega$ with $p>1$. In the previous formula, $\rho$ corresponds to the design function of the considered optimization Problem (10). The reason of using $\rho$ instead of $\eta$ is that typical solutions have values of $\eta$ either close to $\eta_{\min }$ or close to $\eta_{\max }$, with large gradients in between. The change of variable $\eta=\rho^{p}$ allows to deal with solutions easier to be found numerically (see [23]).

Considering the previous notations, minimization Problem (10) can be rewritten as

$$
\begin{aligned}
\min _{\rho} & \frac{\alpha}{\mathbb{E}_{0}} \mathbb{E}[\Psi(\xi, K)]+\frac{\beta}{\operatorname{Var}_{0}} \operatorname{Var}[\Psi(\xi, K)] \\
\text { such that: } & \eta=\rho^{p} \\
& K=\eta K^{0}, \\
& 0 \leq \int_{\Omega} \eta(x) \mathrm{d} x \leq V_{\max } \text { and } \eta: \Omega \rightarrow\left[\eta_{\min }, \eta_{\max }\right],
\end{aligned}
$$

where $\alpha, \beta \in[0,1]$; and $\mathbb{E}_{0}, \operatorname{Var}_{0} \in \mathbb{R}$. The importance of the role of $\alpha$ and $\beta$ and their impact on the solution is discussed in Section 4.2. Additionally, $K^{0}$ represents the fourth-order tensor of a linear elastic isotropic material, satisfying for all $u, v \in\left[H^{1}(\Omega)\right]^{d}$

$$
e(v): K^{0} e(u):=2 \mu e(u) e(v)+\lambda \operatorname{div} u \operatorname{div} v,
$$

where $A B=\sum_{i, j=1}^{d} a_{i j} b_{i j}=\operatorname{Tr}\left(A B^{T}\right)$; and $\mu, \lambda$ are the Lamé constants of the material (see e.g. $[7,1]$ ).

However, the proposed definition of the set of admissible tensors in Problem (24) does not prevent that optimized solutions exhibit microstructures with periodic variation densities. As proposed in the literature (see, e.g. [2]), we tackle this problem indirectly by introducing a computational procedure in the numerical implementation of the minimization Problem (24) presented in the next section.

\section{Numerical implementation of the model}

In order to obtain a numerical solution of Problem (24), for a finite representative set of values for $(\alpha, \beta)$ denoted by $\Sigma$, we consider the methodology described below.

To approximate the solution of System (1), we consider a finite element method (FEM), similar to the one proposed in [23]. Regarding the considered 
benchmark examples presented in Section 4.1, we assume that $\Omega=\left[0, L_{X}\right] \times$ $\left[0, L_{Y}\right]$ for $2 \mathrm{D}$ cases and $\Omega=\left[0, L_{X}\right] \times\left[0, L_{Y}\right] \times\left[0, L_{Z}\right]$ for $3 \mathrm{D}$ cases. Moreover, we consider rectangular finite elements in the $2 \mathrm{D}$ cases and octahedral finite elements in the $3 \mathrm{D}$ case, built by considering a uniform mesh on $\Omega$ of step size $\Delta_{X}, \Delta_{Y}$ and $\Delta_{Z}$ in the $\mathrm{X}, \mathrm{Y}$ and $\mathrm{Z}$ directions (the $\mathrm{Z}$ direction only applies in 3D cases), respectively. Furthermore, we denote by $M$ the total number of finite elements and by $\Theta$ the set of the considered $M$ finite elements. We note that in [23] and [24] the implementation of the 2D case and 3D case are detailed, respectively. Following the standard finite element methodology, with that mesh we obtain a finite dimensional space $V_{h}$ approximating the infinite dimensional space $H$, with $N=\operatorname{dim} V_{\mathrm{h}}$. Then, the solution $u \in H$ of (4) can be approximated by a function $u_{n} \in V_{h}$ whose vector $\{u\} \in \mathbb{R}^{N}$ of coordinates in the nodal base is solution of $[K]\{u\}=\{f\}$, where $[K] \in$ $\mathbb{R}^{N \times N}$ is the stiffness matrix approximating tensor $K$ and $\{f\} \in \mathbb{R}^{N}$ is the load vector containing the coordinates in the nodal base of a function in $V_{h}$ approximating the external forces $f$ and $g$. Matrix $[K]$ can be written as

$$
[K]=\sum_{e \in \Theta}\left[K_{e}\right]
$$

where $\left[K_{e}\right] \in \mathbb{R}^{N \times N}$ is the part of the stiffness matrix corresponding to element $e \in \Theta$. Then, we approximate a density function $\eta: \rightarrow\left[\eta_{\min }, \eta_{\max }\right]$ by a vector $\eta_{e} \in\left[\eta_{\min }, \eta_{\max }\right]^{M}$ representing the piecewise constant function $\bar{\eta}: \Omega \rightarrow\left[\eta_{\min }, \eta_{\max }\right]$ given by $\bar{\eta}(x)=\eta_{e}$, if $x \in e(e \in \Theta)$.

We consider this FEM approach and a structure submitted to one main load (not necessarily a point load, but also, a surface load or a volume load) and $N p$ independent random functions following a normal distribution $\mathcal{N}\left(0, \sigma_{i}^{2}\right)$ or a uniform distribution $U\left(-\sigma_{i}, \sigma_{i}\right)$, for $i=1 \ldots N_{p}$, such as in the examples considered in Sections 4.1.1 and 4.1.3. Then, we discretize directly $\mathbb{E}[\Psi(\xi, K)]$ and $\operatorname{Var}[\Psi(\xi, K)]$ (and not $\Psi(\xi, K)$ as done in $[15,16,17,18]$ ), by considering their explicit expression given in Theorems 1 and 2, and obtain 
that Problem (24) can be approximated by

$$
\begin{aligned}
\min _{\rho} J_{\mathrm{opt}}(\rho)= & \frac{\alpha}{\mathbb{E}_{0}}\left(\sum_{e \in \Theta} u_{e}^{T} K_{e} u_{e}+c_{1} \sum_{i=1}^{N p} \sigma_{i}^{2}\left(\sum_{e \in \Theta} U_{i, e}^{T} K_{e} U_{i, e}\right)\right) \\
& +\frac{\beta}{\operatorname{Var}_{0}}\left(c_{1} \sum_{i=1}^{N p} \sigma_{i}^{2}\left(\sum_{e \in \Theta} u_{e}^{T} K_{e} U_{i, e}\right)^{2}\right. \\
& \left.+c_{2} \sum_{i=1}^{N p} \sigma_{i}^{2} \sum_{j=1}^{N p} \sigma_{j}^{2}\left(\left(\sum_{e \in \Theta} U_{j, e}^{T} K_{e} U_{i, e}\right)\left(\sum_{e \in \Theta} U_{i, e}^{T} K_{e} U_{j, e}\right)\right)\right)
\end{aligned}
$$

such that : $\quad \rho=\left\{\rho_{e}\right\}_{e \in \Theta}$ and $\rho_{e} \in\left[\eta_{\min }^{\frac{1}{p}}, \eta_{\max }^{\frac{1}{p}}\right], \forall e \in \Theta$,

$$
\begin{aligned}
& K_{e}=\rho_{e}^{p} K^{0}, \\
& 0 \leq \sum_{e \in \Theta} \rho_{e}^{p}|e| \leq V_{\max },
\end{aligned}
$$

where $\rho_{e}$ denotes the design function value at finite element $e \in \Theta ;|e|$ the volume or area of element $e$, according to the dimension 2 or 3 of the considered design problem; $K^{0}$ denotes the element stiffness matrix for the reference material at element $e$ expressed in local coordinates (i.e., if $d=2$ then $K_{e} \in \mathbb{R}^{8 \times 8}$ and if $d=3$ then $K_{e} \in \mathbb{R}^{24 \times 24}$, see $\left.[23,24]\right) ; u_{e}$ is the vector of the deformations generated by the main load at the nodes of element $e$ expressed in local coordinates (i.e., if $d=2$ then $u_{e} \in \mathbb{R}^{8}$ and if $d=3$ then $u_{e} \in \mathbb{R}^{24}$ ); $U_{i, e}$ is the vector of the deformations generated by the $i$-th perturbation load at the nodes of element $e$ expressed in local coordinates. Furthermore,

- if the perturbation loads law is $\mathcal{N}\left(0, \sigma_{i}^{2}\right)$, for $i=1 \ldots N_{p}: c_{1}=1$ and $c_{2}=2$,

- if the perturbation loads law is $U\left(-\sigma_{i}, \sigma_{i}\right)$, for $i=1 \ldots N_{p}: c_{1}=\frac{1}{3}$ and $c_{2}=\frac{4}{45}$.

Remark 7. Problem (26) can be also adapted to the cases of random functions following a distribution different from $\mathcal{N}\left(0, \sigma^{2}\right)$ and $U(-\sigma, \sigma)$.

Remark 8. Problem (26) can be generalized to the case of $N_{p}$ dependent random functions. An easy case with dependent random variables is when 
we have $N_{p}$ dependent random functions $\xi_{i}=\varepsilon P_{i}$, where $P_{i} \in\left[L^{2}(\Omega)\right]^{d}$ for $i=1, \ldots, N_{p}$, and $\varepsilon$ is a real random variable following $\mathcal{N}\left(0, \sigma^{2}\right)$ or $U(-\sigma, \sigma)$, such as in the benchmark problems considered in Sections 4.1.2 and 4.1.4. In that case, Problem (24) can be approximated by considering the formulation (26) applied to a unique random function $\xi=\varepsilon \cdot\left(\sum_{i=1}^{N_{p}} P_{i}\right)$.

Optimization Problem (26) is solved by using the Matlab package Global Optimization Platform (freely available at http://www.mat.ucm.es/momat/ software.htm), where $N_{\mathrm{OCM}} \in \mathbb{N}$ iterations of the optimal criteria method (OCM) described in [2] are used as the core algorithm and the initial condition is generated by using $N_{\text {sec }} \in \mathbb{N}$ iterations of a multi-layer secant method. The algorithm runs until the completion of all iterations. A complete description and validation of this methodology can be found in [10, 25, 21]. Here, we only give a brief description of the considered OCM. The evolution of the value of $\rho_{e}$ from iteration $i$ up to iteration $i+1$ of the OCM is governed by:

$$
\rho_{e}^{i+1}= \begin{cases}\max \left(\rho_{\min }, \rho_{e}^{i}-m\right) & , \text { if } \sqrt{B_{e}} \rho_{e}^{i} \leq \max \left(\rho_{\min }, \rho_{e}^{i}-m\right), \\ \min \left(\rho_{\max }, \rho_{e}^{i}+m\right) & , \text { if } \sqrt{B_{e}} \rho_{e}^{i} \geq \min \left(\rho_{\max }, \rho_{e}^{i}+m\right), \\ \sqrt{B_{e}} \rho_{e}^{i} & , \text { else }\end{cases}
$$

where $m \in \mathbb{R}^{+}$is a positive move-limit (i.e., a value that limits the evolution of $\rho_{e}^{i+1}$ from $\left.\rho_{e}^{i}\right) ; B_{e}=\left(-\frac{\widehat{\partial J_{\mathrm{opt}}}}{\partial \rho_{e}}\left(\rho^{i}\right)\right) /\left(l \frac{\partial V\left(\rho^{i}\right)}{\partial \rho_{e}}\right) ; \rho^{i}=\left(\rho_{e}^{i}\right)_{e \in \Theta} ; V\left(\rho^{i}\right)=$ $\sum_{e \in \Theta} \rho_{e}^{i} V_{e}$ is the material volume; $l$ is a Lagrangian multiplier that can be found by a bi-sectioning algorithm; and $\frac{\widehat{\partial J_{\mathrm{opt}}}}{\partial \rho_{e}}\left(\rho^{i}\right)$ is the mesh-independent filtered value of $\frac{\partial J_{\mathrm{opt}}}{\partial \rho_{e}}\left(\rho^{i}\right)$, proposed in [26].

More precisely, as mentioned in Section 2.2, this filter is a numerical technique used to prevent microstructures with periodic variation of densities in the numerical solutions (considering a FEM approach this phenomena is called check-board solutions, see [2]). To this end, $\frac{\widehat{\partial J_{\mathrm{opt}}}}{\partial \rho_{e}}\left(\rho^{i}\right)$ is computed by considering a weighted average of $\frac{\partial J_{\mathrm{opt}}}{\partial \rho_{m}}\left(\rho^{i}\right)$, for elements $m$ which are in the ball $B\left(e, r_{\min }\right)$ of radius $r_{\min }$ centered at the center of mass of element $e$, as following:

$$
\frac{\widehat{\partial J_{\mathrm{opt}}}}{\partial \rho_{e}}\left(\rho^{i}\right)=\sum_{m \subset B\left(e, r_{\min }\right)} \frac{\rho_{m}\left(r_{\min }-\operatorname{dist}(e, m)\right)}{\rho_{e} \sum_{n \subset B\left(e, r_{\min }\right)}\left(r_{\min }-\operatorname{dist}(e, n)\right)} \frac{\partial J_{\mathrm{opt}}}{\partial \rho_{m}}\left(\rho^{i}\right),
$$


where $\operatorname{dist}(a, b)$ is the euclidean distance between the centers of mass of elements $a$ and $b$; and $\frac{\partial J_{\mathrm{opt}}}{\partial \rho_{e}}\left(\rho^{i}\right)$ is calculated as (see [2]-Chapter 1.2.3)

$$
\begin{aligned}
\frac{\partial J_{\mathrm{opt}}}{\partial \rho_{e}}\left(\rho^{i}\right)= & p \rho_{e}^{p-1}\left(\frac{\alpha}{\mathbb{E}_{0}}\left(-u_{e}^{T} K_{e} u_{e}-c_{1} \sum_{i=1}^{N p} \sigma_{i}^{2}\left(U_{i, e}^{T} K_{e} U_{i, e}\right)\right)\right. \\
& -\frac{2 \cdot \beta}{\operatorname{Var}_{0}}\left(c_{1} \sum_{i=1}^{N p} \sigma_{i}^{2}\left(u_{e}^{T} K_{e} U_{i, e} \sum_{m \in \Theta} u_{m}^{T} K_{m} U_{i, m}\right)\right. \\
& \left.\left.+c_{2} \sum_{i=1}^{N p} \sigma_{i}^{2} \sum_{j=i}^{N p} \sigma_{j}^{2}\left(U_{i, e}^{T} K_{e} U_{j, e} \sum_{m \in \Theta} U_{j, m}^{T} K_{m} U_{i, m}\right)\right)\right) .
\end{aligned}
$$

More details about the considered OCM can be found in $[2,23]$.

The solution of Problem (26), with fixed values of $(\alpha, \beta)$, given by our optimization approach is denoted by $\rho_{(\alpha, \beta)}$ and the associated density by $\eta_{(\alpha, \beta)}=\rho_{(\alpha, \beta)}^{p}$.

\section{Numerical examples}

In this Section, we present numerical experiments used to study the behavior of the Variance-Expected compliance model proposed previously. To this aim, in Section 4.1, we detail the considered benchmark structures, the coefficients and the parameters used by the model and the optimization problem. Then, in Section 4.2, we show and analyze the results given by our methodology.

\subsection{Considered benchmark problems}

In this work, Problem (26) is solved by considering four particular sets of benchmark problems, each one focusing on the study of one specific behavior of our model. The first set of experiments, described in Section 4.1.1, is proposed to analyze the impact of the model coefficients $(\alpha ; \beta)$ on the design of solutions robust to main loads and their perturbations. The goal of the second set, detailed in Section 4.1.2, is to present the influence on the solution of the law used to describe the random loads perturbations. Then, with the third set, presented in Section 4.1.3, we check the effect on the solution of variations of the perturbation variance. Next, the objective of the last set of problems, introduced in Section 4.1.4, is to compare the results given by our approach when considering dependent or independent perturbations. 
Finally, in Section 4.1.5, we give the values of the parameters of the numerical model (presented in Section 3) used to approximate the solutions of the benchmark problems presented in Sections 4.1.1-4.1.4. We also detail the outputs considered to analyze the behavior of the solutions obtained by our approach.

Remark 9. We note that the list of numerical problems studied in this work is not fully exhaustive. The proposed experiments are used to give some preliminary results on the behavior of our model. As said in Section 5, a more extensive numerical analysis could be performed to complete the conclusions presented here.

\subsubsection{Robustness of structures regarding $(\alpha ; \beta)$}

Here, we are interested in studying the role of the coefficients $(\alpha ; \beta)$ in the design of solutions resilient both to the main loads and its possible perturbations. Indeed, we have previously observed in [10] that, for a similar model applied to truss structures (which can be considered as a discrete version of the problems solved here), a reasonable ratio between the expected and variance values of the compliance should be considered in order to build structures improved with respect to those obtained with the expected compliance model. By improvement, we mean that we are interested in designing structures with a low compliance value for the main load and with a reduced risk of high compliance in cases of high perturbations scenarios. A phenomenon of mass transfer between trusses supporting the main and perturbed loads was reported. Considering topology optimization, similar studies have been performed in $[18,15]$, but with a different modeling approach (as explained in Section 1) and without the analysis of mass transfer phenomenon and risk of high compliance scenarios. Thus, we want to check if, as expected, a similar behavior to the one observed for trusses is observed for continuous structures.

To this end, we consider two particular benchmark structures, presented below, in both $2 \mathrm{D}$ and $3 \mathrm{D}$ versions. The interest of the $2 \mathrm{D}$ approach is to obtain a fine approximation of the solution whereas the 3D case allows to consider an additional perturbation direction and its impact on the structure. Those benchmark cases have been considered as they are some of the most representative ones used in the literature $[2,16,15]$. Furthermore, they were also studied in our previous works considering the truss framework [8, 10]. Thus, we can perform a direct comparison between the results found here and those presented in the literature. More precisely, we consider: 
a) Michel: This structure, studied in [2, 8, 10], is a horizontal square cantilever and supports a vertical load.

Considering the $2 \mathrm{D}$ version, we study a domain $\Omega_{2 D, M}=[0,250] \times$ $[0,250]$. The side $\{0\} \times[0,250]$ is assumed fixed. $V_{\max }$ is set to 25000 . A point load $g=(0,-1)$ and a random load $(\varepsilon, 0)$, with $\varepsilon \sim \mathcal{N}(0,1)$, are applied at node $(250,0)$ (i.e. load $g$ is identified by the node $(250,0)$ and the vector $(0,-1)$ so that $\int_{\Gamma_{g}} g \cdot v \mathrm{~d} x=(0,-1) \cdot v(250,0), \forall v \in H$. We do not repeat this explanation in the following cases). This problem is denoted by MICH2D.

In the $3 \mathrm{D}$ problem, the structure is defined by the domain $\Omega_{3 D, M}=$ $[0,20] \times[0,20] \times[0,20]$. The face $\{0\} \times[0,20] \times[0,20]$ is fixed and $V_{\max }=$ 2400. A main point load $g=(0,0,-1)$ is applied at the node $(20,10,0)$. At the same node, we consider a random point load $\xi=\varepsilon_{1} P_{1}+\varepsilon_{2} P_{2}$, with $\varepsilon_{1}$ and $\varepsilon_{2}$ following $\mathcal{N}(0,1), P_{1}=(1,0,0)$ and $P_{2}=(0,1,0)$. This problem is denoted by MICH3D.

b) Dome: This structure, also considered in $[8,10,16,15]$, is a vertical square that supports a vertical load.

In the $2 \mathrm{D}$ case, we consider a domain $\Omega_{2 D, D}=[0,250] \times[0,250]$ and the side $[0,250] \times\{0\}$ is fixed. $V_{\max }$ is set to 25000 . A point load $g=(0,-1)$ and a random load $(\varepsilon, 0)$, with $\varepsilon \sim \mathcal{N}(0,1)$, are applied at node $(125,250)$. This problem is denoted by DOME2D.

The $3 \mathrm{D}$ structure is the domain $\Omega_{3 D, D}=[0,20] \times[0,20] \times[0,20]$. The face $[0,20] \times[0,20] \times\{0\}$ is fixed and $V_{\max }=2400$. A point load $g=(0,0,-1)$ is applied at the node $(10,10,20)$. Furthermore, at this node we consider a random point load $\xi=\varepsilon_{1} P_{1}+\varepsilon_{2} P_{2}$, with $\varepsilon_{1}$ and $\varepsilon_{2}$ following $\mathcal{N}(0,1), P_{1}=(1,0,0)$ and $P_{2}=(0,1,0)$. This problem is denoted by DOME3D.

A geometrical representation of the $2 \mathrm{D}$ and $3 \mathrm{D}$ domains of the benchmark problems explained above is given in Figure 2.

The solution of Problem (26), with fixed values of $(\alpha, \beta)$, given by our optimization approach is denoted by $\rho_{(\alpha, \beta)}$ and the associated density by $\eta_{(\alpha, \beta)}=\rho_{(\alpha, \beta)}^{p}$.

\subsubsection{Law of the perturbation}

As observed in [15], where the authors apply the model presented in [16], the law of the perturbations of the loads may have an important impact on 


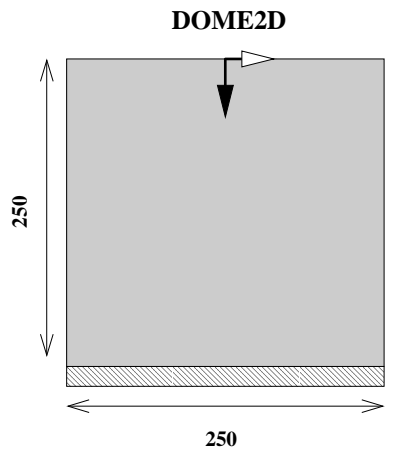

HOBR2D

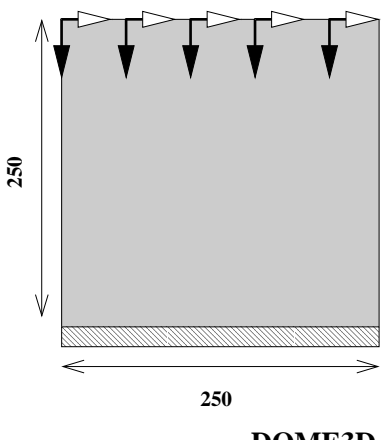

20
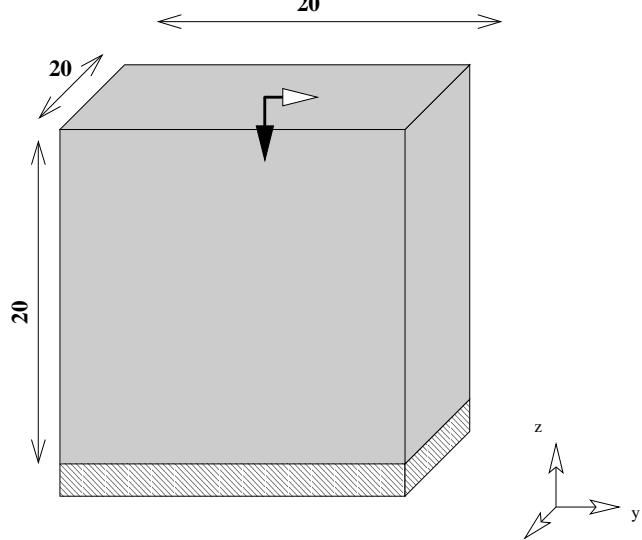

Legend
MICH2D

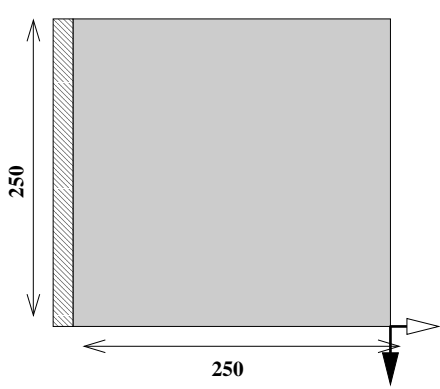

CLDP2D

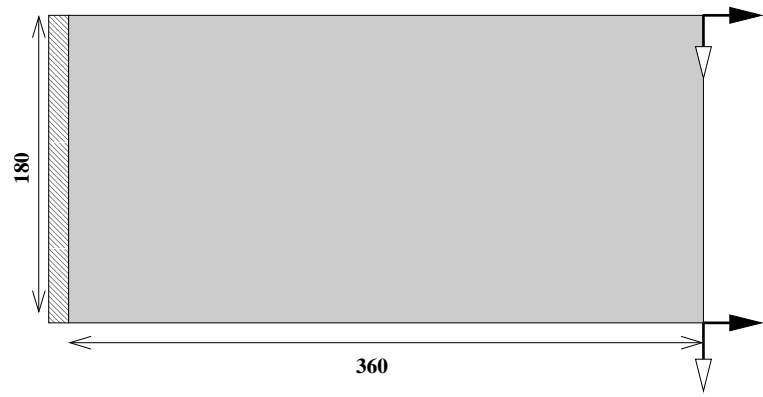

MICH3D
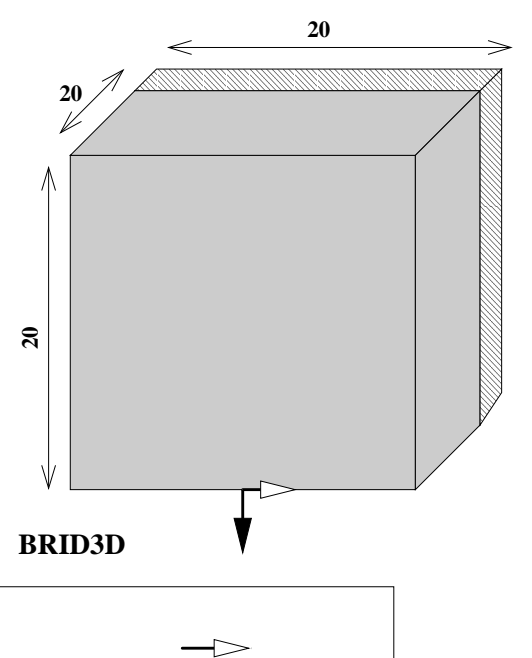

Design domain Support boundary Main load direction Perturbed load direction

Figure 2: Geometrical representation of the 2D benchmark problems MICH2D, DOME2D, HOBR2D and CLDP2D; and the 3D benchmark problems MICH3D and DOME3D. 
the topology of the optimized solution. However, in the example proposed in [15] the differences between the structures generated by considering three particular law distributions (Normal, Uniform and Gumbel) are very slight.

Here, we want to reproduce a similar study by using our model. To this aim, we consider a new benchmark problem, similar to the one used in [15], whose domain is $\Omega_{2 D, H}=[0,250] \times[0,250]$ and such that the side $[0,250] \times\{0\}$ is fixed. $V_{\max }$ is set to 25000 . We assume we have a surface load that can be approximated by a load $g=(0,-1)$ applied to all nodes with coordinates $(x, 250)$, with $x \in \mathbb{N} \cap[0,250]$. This general problem is denoted by HOBR2D and is represented by Figure 2. We consider two problems:

- HOBR2D-N: random loads $(\varepsilon, 0)$ with $\varepsilon$ following a normal law $\mathcal{N}(0$, $0.25)$ are applied to the points with coordinates $(x, 250)$, with $x \in$ $\mathbb{N} \cap[0,250]$.

- HOBR2D-U: random loads $(\varepsilon, 0)$ with $\varepsilon$ following an uniform law $\mathcal{U}(-0.866,0.866)$ are applied to the points with coordinates $(x, 250)$, with $x \in \mathbb{N} \cap[0,250]$.

For HOBR2D-N, the solution of Problem (26) when considering a particular value for $(\alpha, \beta)$, is denoted by $\rho_{(\alpha, \beta)}^{N}$ and the associated density by $\eta_{(\alpha, \beta)}^{N}=$ $\left(\rho_{(\alpha, \beta)}^{N}\right)^{p}$. For HOBR2D-U, they are denoted by $\rho_{(\alpha, \beta)}^{U}$ and $\eta_{(\alpha, \beta)}^{U}$, respectively.

\subsubsection{Variance of the perturbation}

Now, we focus on the study of the impact of possible changes in the variance of the loads perturbations on the solution. As reported in [15], significant modifications on the shape of the solution have been observed by considering the DOME2D structure, presented in Section 4.1.1, and the model introduced in [16].

To carry out a similar analysis with our approach, we consider the DOME-2D benchmark case by replacing the law of the load perturbation by $\mathcal{N}\left(0, \sigma^{2}\right)$, with $\sigma^{2}=0.5,2$ and 5. For each value of $\sigma$, the solution of Problem (26) when considering a particular value for $(\alpha, \beta)$, is denoted by $\rho_{(\alpha, \beta)}^{\sigma}$ and the associated density by $\eta_{(\alpha, \beta)}^{\sigma}=\left(\rho_{(\alpha, \beta)}^{\sigma}\right)^{p}$. This problem is denoted by DOME2D-A.

\subsubsection{Dependence of the perturbations}

As said in Section 2, one of the main advantage of our model regarding the one presented in $[16,18]$ is that it allows to design structures submitted 
to dependent random perturbations. To illustrate the impact on the solution of considering independent or dependent variables, we study two new benchmark problems. More precisely, we consider a $2 \mathrm{D}$ structure, similar to the one presented in [8], whose domain is $\Omega_{2 D},=[0,360] \times[0,180]$. The side $\{0\} \times[0,180]$ is fixed. $V_{\max }$ is set to 25920 . Two main loads $g_{1}=(1,0)$ and $g_{2}=(1,0)$ are applied to the nodes with coordinates $(360,0)$ and $(360,180)$, respectively. This general problem is denoted by CLDP2D and is depicted by Figure 2. Thus, we consider two sub-cases:

- CLDP2D-I: two independents random loads $\left(\varepsilon_{1}, 0\right)$ and $\left(\varepsilon_{2}, 0\right)$, with $\varepsilon_{1}$ and $\varepsilon_{2}$ following a law $\mathcal{N}(0,1)$ are applied to the points with coordinates $(360,0)$ and $(360,180)$.

- CLDP2D-D: two dependent random loads $\left(\varepsilon_{1}, 0\right)$ and $\left(\varepsilon_{2}, 0\right)$, with $\varepsilon_{1}=\varepsilon_{2}$ following a law $\mathcal{N}(0,1)$ are applied to the points with coordinates $(360,0)$ and $(360,180)$. In this case, we have that $\operatorname{Cov}\left(\varepsilon_{1}, \varepsilon_{2}\right)=1$.

For CLDP2D-I, the solution of Problem (26) when considering a particular value for $(\alpha, \beta)$, is denoted by $\rho_{(\alpha, \beta)}^{I}$ and the associated density by $\eta_{(\alpha, \beta)}^{I}=$ $\left(\rho_{(\alpha, \beta)}^{I}\right)^{p}$. For CLDP2D-D, they are denoted by $\rho_{(\alpha, \beta)}^{D}$ and $\eta_{(\alpha, \beta)}^{D}$, respectively.

\subsubsection{Numerical resolution}

The solutions of the benchmark problems presented in Sections 4.1.1-4.1.4 are approximated by considering the numerical implementation described in Section 3 and the representative values $(\alpha, \beta) \in \Sigma:=\{(1,0),(0.75,0.25)$, $(0.5,0.5),(0.25,0.75),(0,1)\}$ (see $[10,11])$. We set $\Delta_{X}=\Delta_{Y}=\Delta_{Z}=1$, $\eta_{\min }=10^{-3}, \eta_{\max }=1, \lambda=0.58, \mu=0.38, r_{\min }=1.5$ and $p=3$ (see [23]); $N_{\mathrm{OCM}}=500$ and $N_{\mathrm{sec}}=5$ (see [10]). Furthermore, the reference coefficients $\mathbb{E}_{0}$ and $\operatorname{Var}_{0}$ used in Equation (24), are set to the expected compliance and variance values of the structure with an uniform density of $\eta=0.4$. This uniform structure is used as the initial point of the optimization algorithm. Values $\mathbb{E}_{0}$ and $\operatorname{Var}_{0}$ for Problems MICH2D, DOME2D, MICH3D, DOME3D, HOBR2D-N, HOBR2D-U and DOME2D-A are given in Table 1.

Solving a $2 \mathrm{D}$ or a 3D case, when considering those parameters with a Matlab 2014a implementation of this numerical model running on a $2.7 \mathrm{Ghz}$ QuadCore CPUs with $16 \mathrm{~Gb}$ of ram, takes around 1h40mins and 2h40mins, respectively. 


\begin{tabular}{cccc}
\hline Problem & MICH2D & DOME2D & MICH3D \\
\hline $\mathbb{E}_{0}$ & 261 & 115 & 45 \\
\hline $\operatorname{Var}_{0}$ & $5.0 \times 10^{4}$ & $1.2 \times 10^{4}$ & 1116 \\
\hline Problem & DOME3D & HOBR2D-N & HOBR2D-U \\
\hline $\mathbb{E}_{0}$ & 21 & $9.2 \times 10^{5}$ & $2.3 \times 10^{6}$ \\
\hline $\operatorname{Var}_{0}$ & 210 & $1.5 \times 10^{12}$ & $9.0 \times 10^{12}$ \\
\hline Problem & DOME2D-0.5 & DOME2D-2 & DOME2D-5 \\
\hline $\mathbb{E}_{0}$ & 46 & 665 & 9858 \\
\hline $\operatorname{Var}_{0}$ & 771 & $2.0 \times 10^{5}$ & $7.8 \times 10^{6}$ \\
\hline
\end{tabular}

Table 1: Values $\mathbb{E}_{0}$ and $\operatorname{Var}_{0}$ for Problems: MICH2D; DOME2D; MICH3D; DOME3D; HOBR2D-N; HOBR2D-U; DOME2D-A with $\sigma^{2}=.5$ (DOME2D-0.5), $\sigma^{2}=2\left(\right.$ DOME2D-2), and $\sigma^{2}=5$ (DOME2D-5).

Moreover, for each case, we also solve numerically the problem of minimizing the compliance value of the structure submitted to the main load $g$ without perturbation load. The density associated to the solution of this last problem is denoted by $\eta_{\text {comp }}$.

For each benchmark problem, in order to have a qualitative comparison of densities $\left\{\eta_{(\alpha, \beta)}\right\}_{(\alpha, \beta) \in \Sigma}$ and $\eta_{\text {comp }}$, we analyze their robustness when they are submitted to random loads and their density distribution. For this purpose, we first compute the compliance value, the expected compliance value and the variance value of each structure. Then, similarly to our previous work [10, 11], we perform a risk analysis of the scenarios generating high compliance values in the structure (called extreme scenarios). To this end, for each $\eta_{(\alpha, \beta)}$, we consider the random variable $\Phi_{\eta_{(\alpha, \beta)}}=\Psi\left(\xi, \eta_{(\alpha, \beta)}\right)$. We approximate the density function of $\Phi_{\eta_{(\alpha, \beta)}}$, denoted by $\gamma_{\Phi_{\eta_{(\alpha, \beta)}}}$, by using a Monte-Carlo approach [21] that generates $M \in \mathbb{N}$ possible scenarios (i.e., values of $\xi$ ). Finally, we calculate two representative statistical values of $\gamma_{\Phi_{\eta_{(\alpha, \beta)}}}$ associated to extreme scenarios: its maximum value and its $\nu \%$-Coherent-Value at Risk $\left(\mathrm{C}-\mathrm{VaR}_{\nu}\right)$.

The $\nu \%$-Coherent-Value at Risk $(\mathrm{C}-\mathrm{VaR})$ is a risk measure defined as:

$$
\mathrm{C}-\operatorname{VaR}_{\nu}(\chi)=\frac{1}{\nu} \int_{0}^{\nu} \inf \left\{z \in \mathbb{R} \text { s.t. } \int_{0}^{z} 100 \nu_{\chi}(x) \mathrm{d} x>(100-y)\right\} \mathrm{d} y,
$$


where $\nu$ is a percentile, $\chi \in L^{\infty}(\mathcal{B}, \mathcal{F}, \mathbb{P}),(\mathcal{B}, \mathcal{F}, \mathbb{P})$ is a probability space, and $\nu_{\chi}$ is the density function of $\chi . \mathrm{C}-\mathrm{VaR}_{\nu}$ corresponds to the average value of the worst $\nu \%$ case scenarios of $\chi$ (i.e., the $\nu \%$ highest values of $\chi$ ). A presentation and an application of $\mathrm{C}-\mathrm{VaR}$ can be found in [21]. In our case, we have $\chi=\Phi_{\eta_{(\alpha, \beta)}}$. Here, we consider $M=1000$ and $\nu=5 \%$ [10].

\subsection{Numerical Results}

We present in Sections 4.2.1-4.2.4 the results obtained by considering the parameters detailed in 4.1.5 and the sets of benchmark cases described in Sections 4.1.1-4.1.4. Only relevant results (i.e., that help to understand the analysis of the behavior of the model) obtained with the numerical experiments are reported. For instance, in Section 4.2.2, the maximum and Coherent Value at Risk values of the compliance are omitted as they are not needed for analyzing the impact of the perturbation law on the structure geometry.

\subsubsection{Robustness of structures regarding $(\alpha ; \beta)$}

The results found with the numerical experiments presented in Section 4.1.1 are summarized in Table 2. The 2D density distributions $\eta_{(\alpha, \beta)}$, when $(\alpha, \beta) \in \Sigma$ for the MICH2D, and DOME2D cases are shown in Figures 3 and 4 , respectively. The $3 \mathrm{D}$ density distributions $\eta_{(\alpha, \beta)}$, when $(\alpha, \beta) \in \Sigma$ for the MICH3D and DOME3D problems are depicted in Figures 5 and 6 , respectively.

As the benchmark cases studied here are quite numerous and the conclusions are similar between them, we will only exhibit some general tendencies regarding the behaviour of our model when considering different values of $\alpha$ and $\beta$.

We can observe in Table 2, that minimizing only the compliance and not considering the expected compliance nor the variance (i.e., $\eta_{\text {comp }}$ ), we obtain structures robust to the main load (i.e., for each problem, $\eta_{\text {comp }}$ shows the lowest compliance value). However, $\eta_{\text {comp }}$ is generally less stable to load perturbations than the structures from $\eta(1,0)$ up to $\eta_{(0.25,0.75)}$, as its Maximum and $\mathrm{C}-\mathrm{VaR}_{5}$ compliance values estimated with the Monte-Carlo approach are the highest ones. For instance, when considering the DOME2D case, the $\mathrm{C}-\mathrm{VaR}_{5}$ and Maximum compliance values of $\eta_{\text {comp }}$ are 117 and 361, respectively, which represent an increase of $300 \%$ when regarding the $\mathrm{C}-\mathrm{VaR}_{5}$ and Maximum compliance values of $\eta_{(1,0)}$ up to $\eta_{(0.25,0.75)}$. In counterpart, the compliance values of $\eta_{(1,0)}$ up to $\eta_{(0.25,0.75)}$ are only between $20 \%$ and 


\begin{tabular}{|c|c|c|c|c|c|c|}
\hline & $\eta_{\text {comp }}$ & $\eta_{(1,0)}$ & $\eta_{(0.75,0.25)}$ & $\eta_{(0.5,0.5)}$ & $\eta_{(0.25,0.75)}$ & $\eta_{(0,1)}$ \\
\hline \multicolumn{7}{|c|}{ MICH2D } \\
\hline Comp & 13.3 & 13.8 & 14.1 & 14.4 & 20.1 & 38.5 \\
\hline $\mathrm{EC}$ & 24.1 & 22.2 & 22.3 & 22.5 & 27.4 & 46.1 \\
\hline Vari & 385 & 237 & 220 & 219 & 210 & 148 \\
\hline C-VaR 5 & 63.2 & 54.4 & 53.7 & 47.1 & 58.9 & 94.2 \\
\hline Max & 130 & 119 & 108 & 102 & 150 & 215 \\
\hline \multicolumn{7}{|c|}{ DOME2D } \\
\hline Comp & 2.51 & 3.05 & 3.14 & 3.25 & 3.36 & 4.72 \\
\hline $\mathrm{EC}$ & 30.5 & 12.2 & 12.5 & 12.8 & 13.6 & 18.9 \\
\hline Vari & 1560 & 216 & 188 & 175 & 166 & 103 \\
\hline C- $\operatorname{VaR}_{5}$ & 117 & 43.8 & 39.0 & 38.3 & 41.3 & 64.9 \\
\hline Max & 361 & 120 & 87.0 & 86.2 & 119 & 177 \\
\hline \multicolumn{7}{|c|}{ MICH3D } \\
\hline Comp & 1.92 & 2.09 & 2.13 & 2.15 & 2.16 & 4.98 \\
\hline $\mathrm{EC}$ & 6.76 & 5.32 & 5.57 & 5.59 & 5.61 & 8.51 \\
\hline Vari & 27.9 & 14.1 & 14.0 & 13.7 & 12.3 & 10.5 \\
\hline C-VaR 5 & 16.9 & 13.1 & 12.6 & 12.5 & 12.7 & 16.7 \\
\hline Max & 54.7 & 27.2 & 25.7 & 23.8 & 29.0 & 35.5 \\
\hline \multicolumn{7}{|c|}{ DOME3D } \\
\hline Comp & 0.68 & 0.71 & 0.74 & 0.76 & 0.79 & 0.80 \\
\hline EC & 4.32 & 3.06 & 3.12 & 3.18 & 3.23 & 3.41 \\
\hline Vari & 13.1 & 5.34 & 5.27 & 5.15 & 4.81 & 4.72 \\
\hline C-VaR 5 & 10.6 & 7.26 & 7.11 & 7.42 & 7.83 & 7.97 \\
\hline $\operatorname{Max}$ & 32.9 & 19.4 & 18.5 & 19.5 & 19.8 & 21.1 \\
\hline
\end{tabular}

Table 2: Summary of the results obtained considering the densities $\eta_{\text {comp }}, \eta_{(1,0)}, \eta_{(0.75,0.25)}$, $\eta_{(0.5,0.5)}, \eta_{(0.25,0.75)}$ and $\eta_{(0,1)}$ for the Problems MICH2D, BRID3D, MICH3D and BRID3D: Compliance (Comp), Expected compliance (EC), Variance of the compliance (Vari), maximum compliance value (Max) and Coherent Value at Risk of the compliance $\left(\mathrm{C}-\mathrm{VaR}_{5}\right)$. 

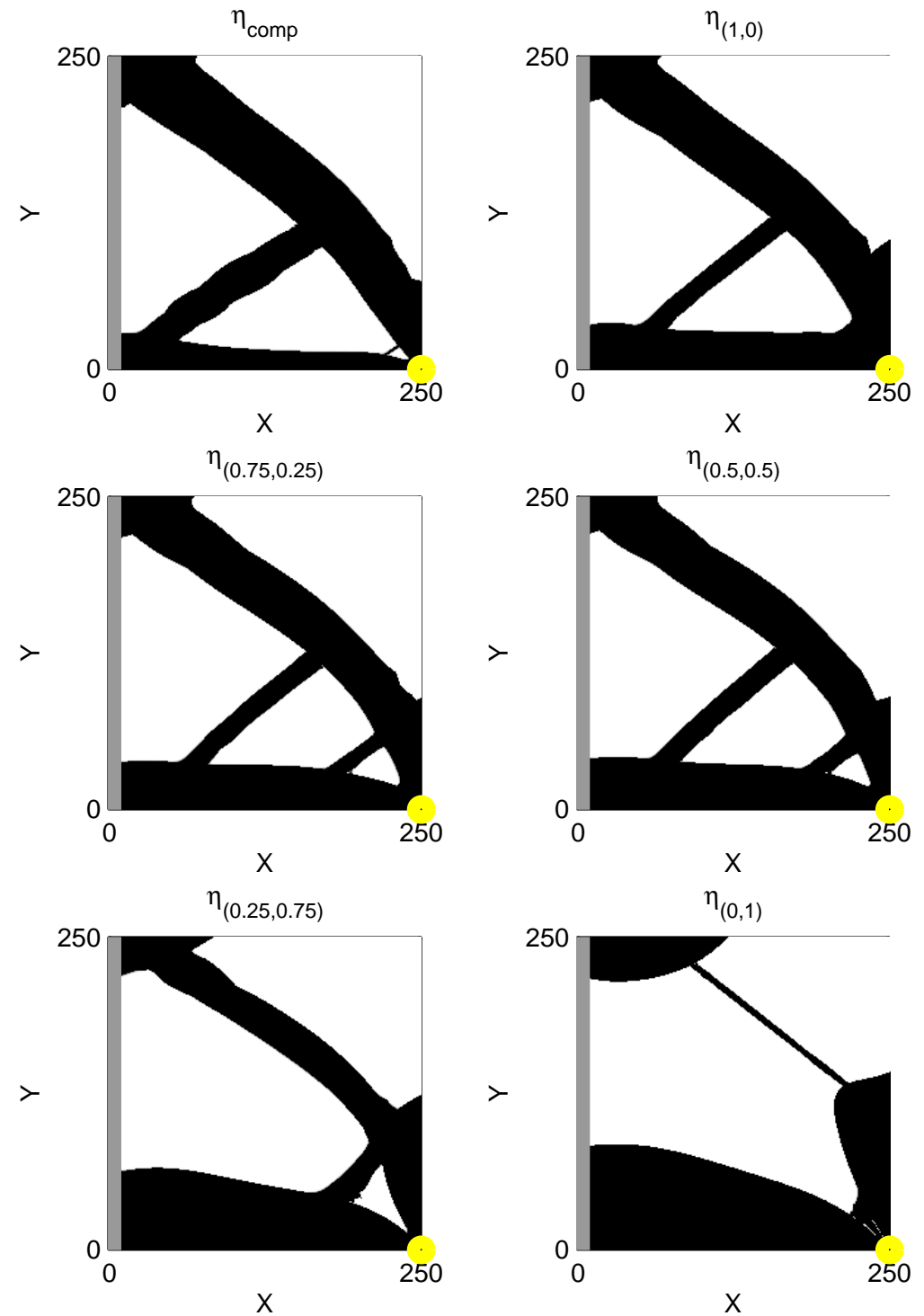

Figure 3: Density distributions $\eta_{\text {comp }}, \eta_{(1,0)}, \eta_{(0.75,0.25)}, \eta_{(0.5,0.5)}, \eta_{(0.25,0.75)}$ and $\eta_{(0,1)}$ obtained for the MICH2D Problem. The support is presented by a grey rectangle at position $x=0$. The node where loads are applied are represented by a bright circle. 


$$
\hat{n} \hat{n}
$$



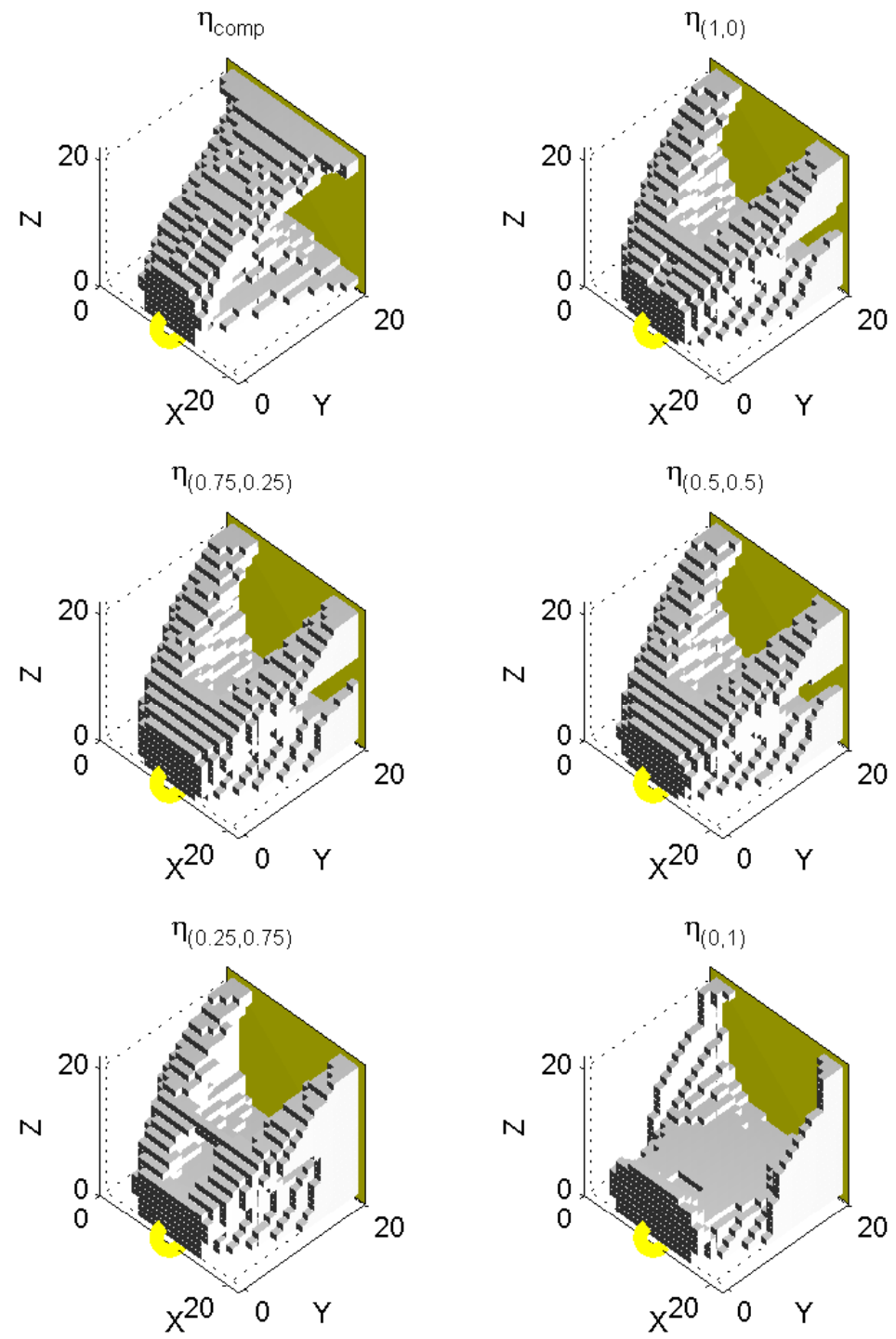

Figure 5: Density distributions $\eta_{\text {comp }}, \eta_{(1,0)}, \eta_{(0.75,0.25)}, \eta_{(0.5,0.5)}, \eta_{(0.25,0.75)}$ and $\eta_{(0,1)}$ obtained for the MICH3D Problem. One support is presented by a filled plane at position $x=0$. The node where loads are applied are represented by a bright circle. 

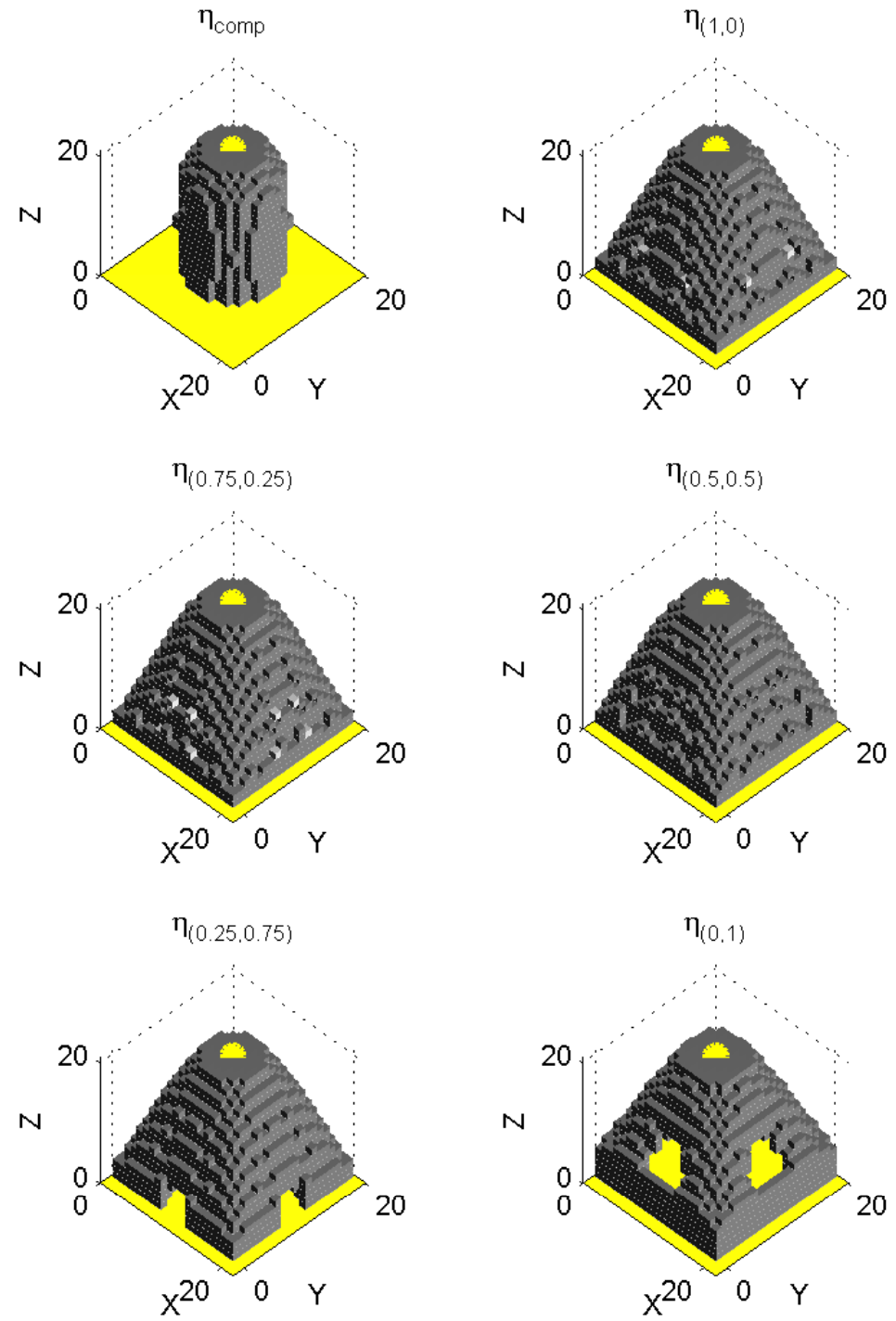

Figure 6: Density distributions $\eta_{\text {comp }}, \eta_{(1,0)}, \eta_{(0.75,0.25)}, \eta_{(0.5,0.5)}, \eta_{(0.25,0.75)}$ and $\eta_{(0,1)}$ obtained for the DOME3D Problem. One of the support walls is presented by a filled plane at position $x=0$. The node where loads are applied are represented by a bright circle. 
$30 \%$ higher than the $\eta_{\text {comp }}$ one. This seems to indicate that considering the Variance-Expected Compliance model should help to generate structures better adapted to perturbed loads scenarios (i.e., which present reduced deformations in cases of severe perturbations) than the classical compliance model. However, the choice of $(\alpha, \beta)$ is important as in some rare cases, the $\eta_{(\alpha, \beta)}$ structure exhibits worst characteristics than $\eta_{\text {comp }}$. For example, in the MICH2D Problem, $\eta_{(0.25,0.75)}$ and $\eta_{(0,1)}$ have higher compliance, C-VaR and Maximum compliance values than $\eta_{\text {comp }}$. Similar phenomenons were also reported in the truss framework in [10] and in the topology cases (but with a different modeling approach) in $[15,18]$.

When comparing the effect of the weight coefficients $(\alpha, \beta)$ on the solutions, we can observe that if our objective is to reduce the extreme scenarios compliance (i.e., reduce the $\mathrm{C}-\mathrm{VaR}_{5}$ or the Maximum compliance values) both values $\alpha$ and $\beta$ should be strictly positive. For instance, for the MICH3D Problem, $\eta_{(0.5,0.5)}$ shows the lowest $\mathrm{C}-\mathrm{VaR}_{5}$ and maximum compliance values whereas, for the DOME3D case, $\eta_{(0.75,0.25)}$ is the most resilient structure to strong perturbations. This seems to indicate that for each particular design problem, if we are interested in managing severe perturbations, a previous study on the values of $(\alpha, \beta)$ should be performed in order to determine their optimal values.

From a general point of view, when we raise $\beta$ the value of the variance of the compliance decreases and the expected compliance increases. Indeed, as observed in the truss case [10], when we increase the variance weight, we increase the robustness of the structure to the perturbed loads whereas it becomes weaker to the main load. This can be observed on the evolution of the density distribution $\eta_{(\alpha, \beta)}$ in function of $(\alpha, \beta)$. More precisely, regarding Figures 3 and 5, for the MICH2D and MICH3D cases, there is a mass transfer phenomena when $\beta$ increases from the diagonal 'bars' (supporting the vertical main load) to the floor of the structure (which offer a good resistance to the perturbed loads in the plane $z=0$ ). Considering the DOME2D and DOME3D cases, there is a material transfer when $\beta$ raises from the upper part of the dome (sustaining the main load) to the 'feet' of the structure (which offer resistance to horizontal perturbations).

The rates of increase/decrease of the expected compliance and variance values according to the weight coefficients $(\alpha, \beta)$ depend on the studied design problem. For instance, on the one hand, for the DOME3D experiment the expected compliance raises $12 \%$ and the variance decreases $12 \%$ when comparing $\eta_{(1,0)}$ with $\eta_{(0,1)}$. On the other hand, for the MICH3D case, the 


\begin{tabular}{ccccccc}
\hline \multicolumn{7}{c}{ HOBR2D-N } \\
\hline Comp $\left(\times 10^{5}\right)$ & 1.07 & 1.12 & 1.25 & 1.34 & 1.57 & 22.5 \\
EC $\left(\times 10^{5}\right)$ & 5.52 & 1.95 & 2.08 & 2.16 & 2.27 & 23.4 \\
Vari $\left(\times 10^{10}\right)$ & 162 & 6.64 & 5.41 & 5.22 & 4.94 & 3.61 \\
\hline \multicolumn{7}{c}{ HOBR2D-U } \\
\hline Comp $\left(\times 10^{5}\right)$ & 1.09 & 1.39 & 1.46 & 1.57 & 1.65 & 27.5 \\
EC $\left(\times 10^{5}\right)$ & 15.6 & 5.08 & 5.14 & 5.35 & 5.56 & 58.0 \\
$\operatorname{Vari}\left(\times 10^{10}\right)$ & 970 & 47.3 & 30.5 & 29.6 & 28.6 & 24.7 \\
\hline
\end{tabular}

Table 3: Summary of the results obtained considering the densities $\eta_{\text {comp }}, \eta_{(1,0)}^{N}, \eta_{(0.75,0.25)}^{N}$, $\eta_{(0.5,0.5)}^{N}, \eta_{(0.25,0.75)}^{N}$ and $\eta_{(0,1)}^{N}$ for Problems HOBR2D-N and Problem HOBR2D-U: Compliance (Comp), Expected compliance (EC) and Variance of the compliance (Vari).

expected compliance is increased by $60 \%$ and the variance decreased by $25 \%$ when considering $\eta_{(1,0)}$ in front of $\eta_{(0,1)}$. As said previously, the choice of $(\alpha, \beta)$ should be adapted to the considered design problem.

From a general point of view, those conclusions are consistent with other similar studies $[10,15,18]$ that show, for modeling techniques different to the one presented here, the interest of considering Variance-Expected compliance terms, with a good ratio between them, to generate structures that are robust to random loads.

\subsubsection{Law of the perturbation}

The results found with the numerical experiments presented in Section 4.1.2 are summarized in Table 3. Some representative density distributions $\eta_{(\alpha, \beta)}^{N}$ and $\eta_{(\alpha, \beta)}^{U}$ for the HOBR2D-N and HOBR2D-U cases are shown in Figure 7.

As we can observe in Table 3, in both cases HOBR2D-N and HOBR2D -U, the proposed methodology allows to improve the expected compliance and variance values according to the parameters $(\alpha, \beta)$. The values reported in the uniform law case are higher than in the normal law one. This result is expected since, on the one hand, considering the uniform law, high perturbations scenarios have the same probability to occur than low perturbations 


\begin{tabular}{c|ccc|ccc|ccc}
\hline & $\eta_{(1,0)}^{0.5}$ & $\eta_{(.5,5)}^{0.5}$ & $\eta_{(0,1)}^{0.5}$ & $\eta_{(1,0)}^{2}$ & $\eta_{(.5,5)}^{2}$ & $\eta_{(0,1)}^{2}$ & $\eta_{(1,0)}^{5}$ & $\eta_{(.5,5)}^{5}$ & $\eta_{(0,1)}^{5}$ \\
\hline $\mathbf{C}$ & 2.92 & 3.01 & 4.31 & 4.17 & 4.24 & 4.95 & 5.88 & 6.56 & 9.87 \\
$\mathbf{E}$ & 4.02 & 4.24 & 7.72 & 78.0 & 78.4 & 81.3 & 1112 & 1139 & 1184 \\
$\mathbf{V}$ & 28.9 & 10.3 & 9.72 & 2956 & 2747 & 2735 & 105011 & 103133 & 100327 \\
\hline
\end{tabular}

Table 4: Summary of the results obtained considering the densities $\eta_{(1,0)}^{\sigma^{2}}, \eta_{(.5, .5)}^{\sigma^{2}}$ and $\eta_{(0,1)}^{\sigma^{2}}$, with $\sigma^{2}=0.5,2$ and 5, for Problem DOME2D-A: Compliance (C), Expected compliance (E) and Variance of the compliance $(\mathbf{V})$.

ones. On the other hand, considering the normal law, small perturbation scenarios have much more probability to occur than other ones.

Regarding Figure 7, we observe that the shape of $\eta^{U}(\alpha, \beta)$ and $\eta^{N}(\alpha, \beta)$, for a fixed value $(\alpha, \beta)$, are quite different. In the uniform law case the two main inclined columns are thicker than the normal law case. Indeed, as said previously, since important perturbation scenarios occur more frequently those two columns offer a better resistance to lateral perturbations.

As said above, in [15] a similar example is also given to show the impact on the structure shape of considering different random laws. However, the results presented in [15] show very slight changes between laws. This difference can be explained by the choice of different mean and variance values of the load perturbations, set to -1.6 and 0.17 , respectively. Indeed, the authors consider a random perturbation which always impact the main load within a small range of variation. In our work we consider perturbations with mean 0 and a stronger variance of 0.25 . However, in both works, results point out the fact that, considering a expected compliance-variance model, the random law used to model the perturbation behavior should be chosen carefully as it impacts the final structure shape.

\subsubsection{Variance of the perturbation}

Here, we study the cases presented in Section 4.1.3. The results are summarized in Table 4. The density distributions of the considered solutions are depicted in Figure 8.

As we can see in Table 4, the values of the compliance and the expected and variance of compliance increase strongly as $\sigma$ is raised. This increase results in changes in the solution geometry of $\eta_{(\alpha, \beta)}^{\sigma^{2}}$, for fixed values of $(\alpha, \beta)$, as observed in Figure 8. For instance, from $\sigma=0.5$ to $\sigma=2$, we see the emergence of diagonal bars that help the structure to support strong lateral 

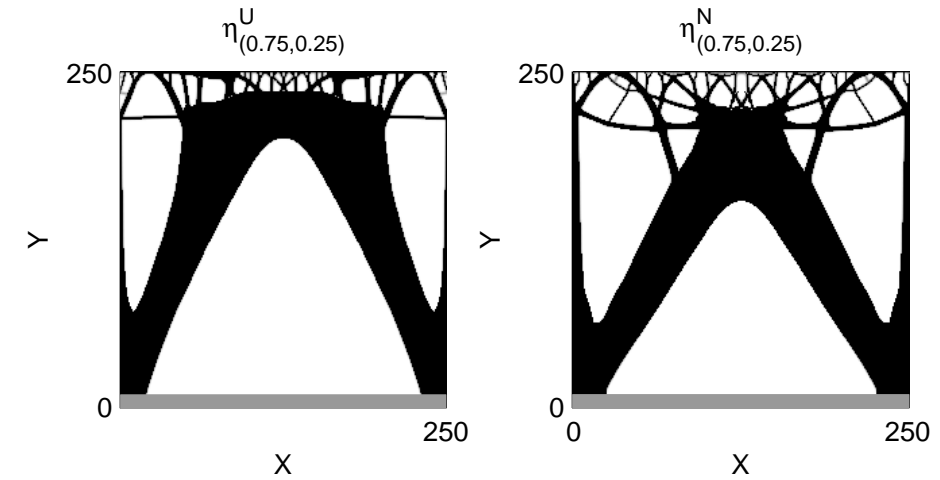

$\eta_{(0.5,0.5)}^{U}$

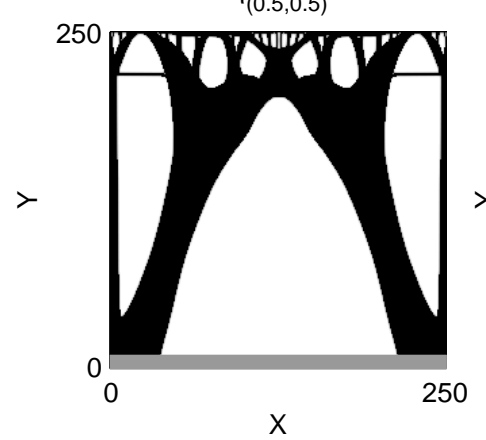

$\eta_{(0.5,0.5)}^{\mathrm{N}}$

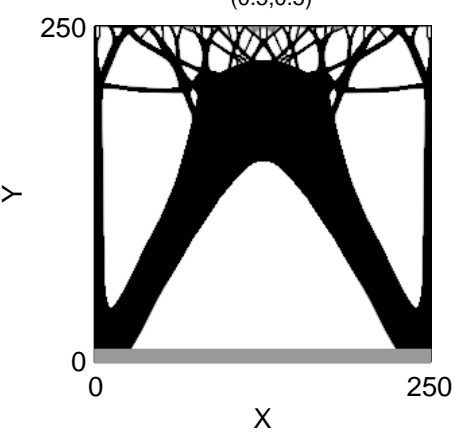

$\eta_{(0.25, .75)}^{U}$

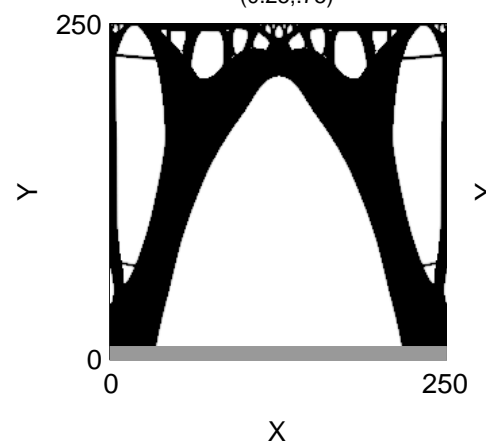

$\eta_{(0.25, .75)}^{\mathrm{N}}$

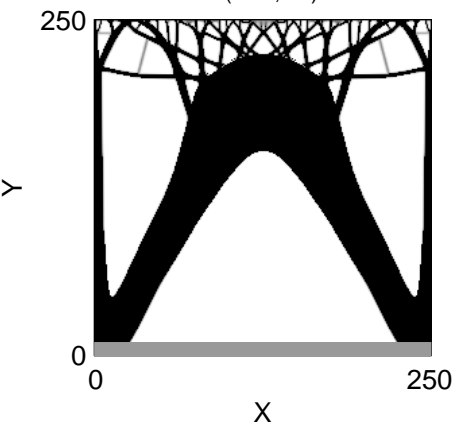

Figure 7: Density distributions: (Left) $\eta_{(0.75,0.25)}^{U}, \eta_{(0.5,0.5)}^{U}$ and $\eta_{(0.25,0.75)}^{U}$ obtained for Problem HOBR2D-U in case of perturbations with uniform distribution; and (Right) $\eta_{(0.75,0.25)}^{N}, \eta_{(0.5,0.5)}^{N}$ and $\eta_{(0.25,0.75)}^{N}$ obtained for Problem HOBR2D-N in case of perturbations with normal distribution. The support wall is the gray bar at position $y=0$. 

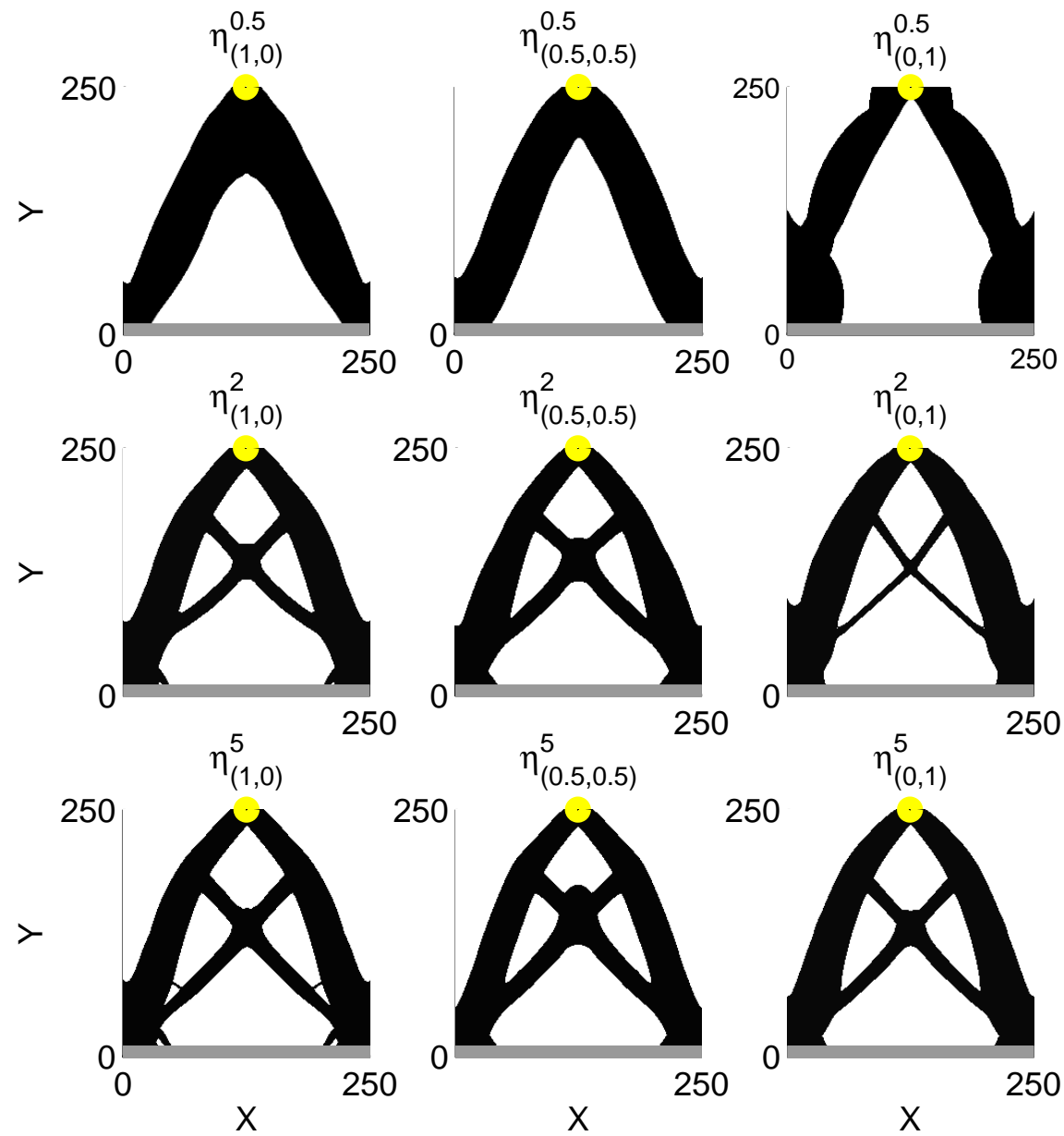

Figure 8: Density distribution $\eta_{(1,0)}^{\sigma^{2}}, \eta_{(0.5,0.5)}^{\sigma^{2}}$ and $\eta_{(0,1)}^{\sigma^{2}}$ obtained for Problem DOME2D with $\sigma^{2}=0.5,2$ and 5. A support wall is presented by a grey rectangle at position $x=0$. The node where loads are applied are represented by a bright circle. 
perturbations. Those bars become thicker from $\sigma=2$ to $\sigma=5$ as the horizontal perturbations variance is raised. We note that, from $\sigma=5$ to $\sigma=10$, changes are very slight (we do not show the results here) which seems to indicate that at a certain value of the perturbation of the variance the structure does not suffer major modifications.

Similar results were also observed in $[15,16]$. Again, due to the considered perturbation characteristics and modeling (in Dunning et al. only the minimization of expected compliance is considered), structure changes in those articles are slighter than in this work. In particular there is no emergence of diagonal bars. In any case, a good estimation of the variance of the perturbation is necessary to generate structures robust to loads and their perturbations.

\subsubsection{Dependence of the perturbations}

Here we study the cases presented in Section 4.1.4. We show in Figure 9 the density distributions of the some relevant solutions of Problems CLDP2D and CLDP2I.

As we can observe in this figure, the topology of the shape solutions changes when considering or not dependence of the random perturbation of loads. Indeed, in the independent case a vertical bar liking the nodes $(360,0)$ and $(360,180)$ appears providing resistance in cases of perturbations occurring in different directions. In the dependent case (here perturbations are the same), this bar does not appear and the other bars are slightly reinforced. This example shows the importance of considering or not dependence in random loads, as this choice affects the final geometry. Furthermore, as said in Section 2, regarding other models in $[15,20,18]$ our approach is better suited to treat such cases.

\section{Conclusions}

In this work, we have presented a Variance-Expected Compliance model to solve stochastic topology optimization problems.

The behavior of this model was studied numerically by considering a finite element implementation and various $2 \mathrm{D}$ and $3 \mathrm{D}$ benchmark cases. In particular, we have studied the impact on the solution characteristics (i.e., compliance thresholds and shape) of (i) the expected value and variance weight coefficients, (ii) the laws used to describe the random loads, (iii) the 

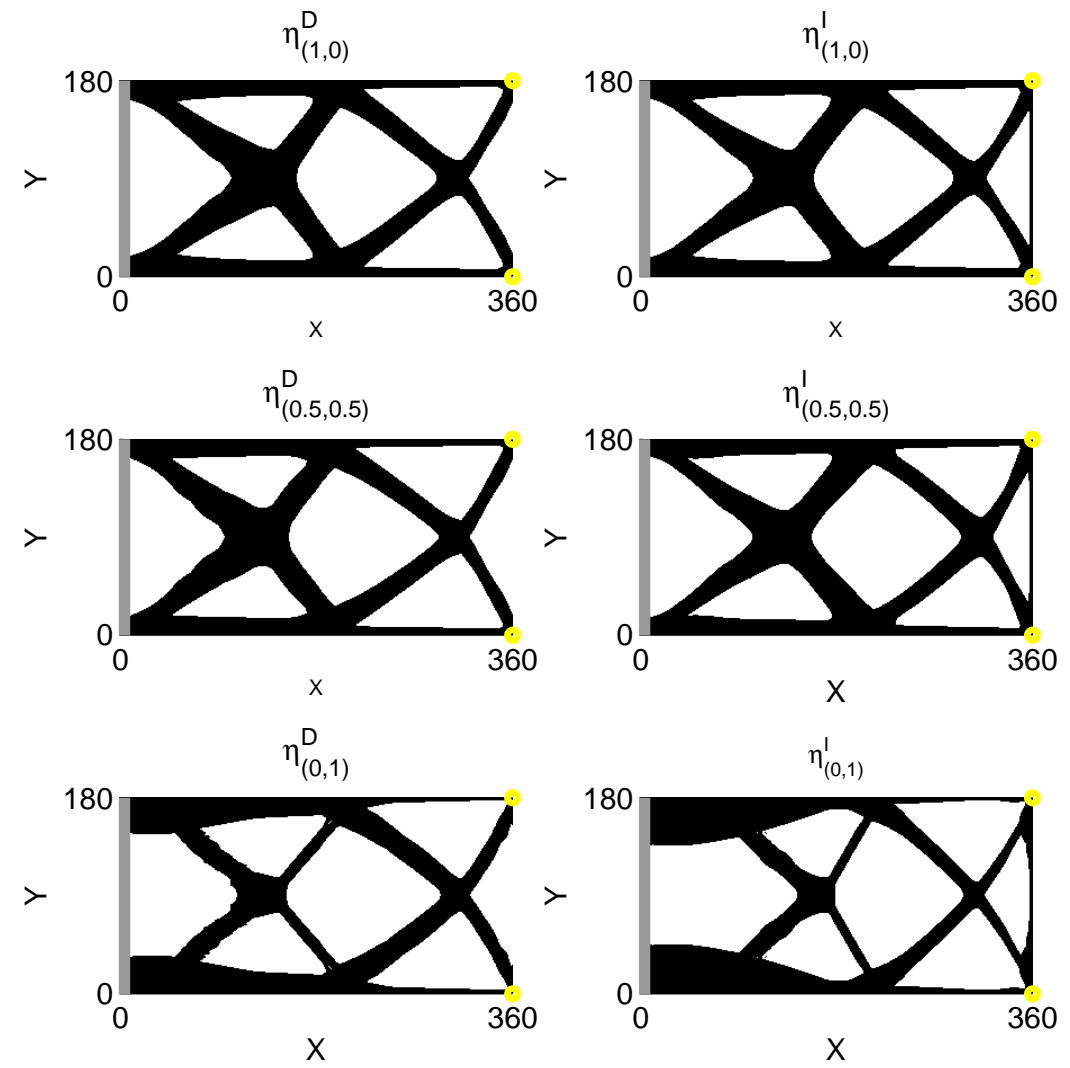

Figure 9: Density distributions: (Left) $\eta_{(1,0)}^{D}, \eta_{(0.5,0.5)}^{D}$ and $\eta_{(0,1)}^{D}$ obtained for Problem CLDP2D in case of two dependent perturbed loads; and (Right) $\eta_{(1,0)}^{I}, \eta_{(0.5,0.5)}^{I}$ and $\eta_{(0,1)}^{I}$ obtained for Problem CLDP2I in case of two independent perturbed loads. A support wall is presented by a gray bar at position $x=0$. The node where loads are applied are represented by a bright circle. 
variation of the perturbation variance and (iv) the dependence or independence of the perturbations. We have seen that considering our approach the solutions given by our model are strongly related to those four characteristics. This was also observed, considering different models for truss or topology optimization, in previous literature (see $[8,10,11,15,20,16,18]$ ). In addition, the obtained results seem to indicate that our formulation with suitable weight coefficients allows to generate structures that are robust to main loads and their perturbations in case of extreme scenarios.

In future works, we will deeply investigate the effect of the dependence of loads perturbations. Moreover, we aim to improve the algorithm proposed here, considering fast and efficient optimization methods. Additionally, we will consider probabilistic constraints in the framework of reliability based optimization design.

\section{Acknowledgments}

This work was carried out thanks to the financial support of the "Universidad de los Andes"; the Spanish "Ministry of Economy and Competitiveness" under project MTM2011-22658; the research group MOMAT (Ref. 910480) supported by "Banco Santander" and "Universidad Complutense de Madrid"; the "Junta de Andalucía" and the European Regional Development Fund under project P12-TIC301; and the "FONDECYT" under grant 1130905.

[1] L. Landau, E. M. Lifshitz, Theory of Elasticity, Oxford, England: Butterworth Heinemann, 1986.

[2] M. Bendsøe, O. Sigmund, Topology Optimization: Theory, Methods and Applications, Springer-Verlag, Berlin, 2003.

[3] M. Bendsøe, Optimization of structural topology, shape, and material, Springer, 1995.

[4] S. Conti, H. Held, M. Pach, M. Rumpf, R. Schultz, Shape optimization under uncertainty, a stochastic programming perspective, SIAM Journal on Optimization 19 (4) (2008) 1610-1632.

[5] J. Alberty, C. Carstensen, S. A. Funken, , R. Klose, Matlab implementation of the finite element method in elasticity, Computing 3 (69) (2002) 269-263. 
[6] P. Ciarlet, The Finite Element Method For Elliptic Problems, NorthHolland, Amsterdam, 1980.

[7] P. Ciarlet, Mathematical Elasticity, Vol. I, Three Dimensional Elasticity., North-Holland, Amsterdam, Amsterdam, 1988.

[8] F. Alvarez, M. Carrasco, Minimization of the expected compliance as an alternative approach to multiload truss optimization, Struct. Multidiscip. Optim. 29 (6) (2005) 470-476.

[9] M. Carrasco, Diseño óptimo de estructuras reticulares en elasticidad lineal vía teoría de la dualidad. estudio teórico y numérico, Ph.D. thesis, Universidad de Chile, engineering Degree Thesis (2003).

[10] M. Carrasco, B. Ivorra, A. M. Ramos, A variance-expected compliance model for structural optimization, J. Optim. Theory Appl. 152 (1) (2012) 136-151.

[11] M. Carrasco, B. Ivorra, R. Lecaros, A. M. Ramos, An expected compliance model for topology optimization, Differ. Equ. Appl 4 (1) (2012) $111-120$.

[12] J. Zhao, C. Wang, Robust topology optimization of structures under loading uncertainty, American Institute of Aeronautics and Astronautics Journal 2 (52) (2014) 398-407.

[13] G. Allaire, C. Dapogny, A linearized approach to worst-case design in parametric and geometric shape optimization, Mathematical Models and Methods in Applied Sciences in press (2014) 1-58.

[14] J. K. Guest, T. Igusa, Structural optimization under uncertain loads and nodal locations, Computer Methods in Applied Mechanics and Engineering 198 (1) (2008) 116 - 124, computational Methods in Optimization Considering Uncertainties.

[15] J. Zhao, C. Wang, Robust topology optimization under loading uncertainty based on linear elastic theory and orthogonal diagonalization of symmetric matrices, Computer Methods in Applied Mechanics and Engineering 273 (1) (2014) $204-218$. 
[16] P. D. Dunning, H. A. Kim, G. Mullineux, Introducing loading uncertainty in topology optimization, American Institute of Aeronautics and Astronautics Journal 49 (4) (2011) 760-768.

[17] P. D. Dunning, H. A. Kim, Robust topology optimization: Minimization of expected and variance of compliance, American Institute of Aeronautics and Astronautics Journal 51 (11) (2014) 2656-2664.

[18] P. D. Dunning, H. A. Kim, Robust topology optimization: Minimization of expected and variance of compliance, American Institute of Aeronautics and Astronautics Journal 51 (11) (2014) 2656-2664.

[19] J. Zhao, C. Wang, Robust structural topology optimization under random field loading uncertainty, Structural and Multidisciplinary Optimization 50 (3) (2014) 517-522.

[20] S. Chen, W. Chen, S. Lee, Level set based robust shape and topology optimization under random field uncertainties, Structural and Multidisciplinary Optimization 41 (4) (2010) 507-524.

[21] B. Ivorra, B. Mohammadi, A. M. Ramos, Optimization strategies in credit portfolio management, Journal Of Global Optimization. 2 (43) (2009) 415-427.

[22] A. M. Ramos, Introducción al análisis matemático del método de elementos finitos, Editorial Complutense, 2012.

[23] O. Sigmund, A 99 line topology optimization code written in matlab, Structural and Multidisciplinary Optimization 2 (21) (2001) 120-127.

[24] K. Liu, A. Tovar, An efficient 3d topology optimization code written in matlab, Structural and Multidisciplinary Optimization (2014) 1-22.

[25] B. Ivorra, D. Hertzog, B. Mohammadi, J. Santiago, Semi-deterministic and genetic algorithms for global optimization of microfluidic proteinfolding devices, International Journal for Numerical Methods in Engineering 66 (2) (2006) 319-333.

[26] O. Sigmund, On the design of compliant mechanisms using topology optimization, Mechanics of Structures and Machines 25 (4) (1997) 493524. 